\title{
LEVANTAMENTO FLORÍSTICO DA FLORESTA SERRANA DA RESERVA BIOLÓGICA DE SERRA NEGRA, MICRORREGIÃO DE ITAPARICA, PERNAMBUCO, BRASIL ${ }^{1}$
}

\author{
Maria Jesus Nogueira Rodal ${ }^{2}$ \\ Ladivania Medeiros do Nascimento ${ }^{3}$
}

Recebido em 17/12/1999. Aceito em 15/05/2002.

\begin{abstract}
RESUMO - (Levantamento florístico da floresta serrana da Reserva Biológica de Serra Negra, microrregião de Itaparica, Pernambuco, Brasil). O levantamento florístico, de duas áreas da Reserva Biológica de Serra Negra, localizada entre os municípios de Inajá e Floresta $\left(8^{\circ} 35^{\prime}-8^{\circ} 38^{\prime}\right.$ S e $38^{\circ} 02^{\prime}-38^{\circ} 04^{\prime}$ W), foi realizado fazendo-se 19 excursões para coleta de material botânico de Bryophyta, Pteridophyta e Magnoliophyta. No total foram coletados 319 taxa, 255 na floresta densa, situada no topo, e 117 na floresta aberta, localizada na escarpa meridional, com 12,5 $\%$ de similaridade (Jaccard). A análise de agrupamento mostrou que as duas áreas têm maiores similaridades com outras florestas serranas da região nordeste. A presença de condições abióticas diferenciadas, úmida no topo e mais seca na escarpa meridional, justifica as diferenças florísticas. Na floresta densa ocorreu um grande número de espécies com ampla distribuição nos domínios amazônico e atlântico, enquanto na floresta aberta parte das espécies foi mais relacionada à vegetação caducifólia espinhosa (caatinga), e outras têm distribuição ampla em florestas neotropicais.
\end{abstract}

Palavras-chave - Floresta serrana, caatinga, florística, semi-árido, nordeste do Brasil

\begin{abstract}
Floristic survey in the montane forest of the Biological Reserve of Serra Negra, Pernambuco, Brazil). A floristic survey was carried out in the Biological Reserve of Serra Negra, between the municipalities of Inajá and Floresta $\left(8^{\circ} 35^{\prime}-8^{\circ} 38^{\prime} \mathrm{S}\right.$ and $\left.38^{\circ} 02^{\prime}-38^{\circ} 04^{\prime} \mathrm{W}\right)$, in order to assess the floristic relationship between two sites, a dense forest on the plateau, and an open forest, located on the southern scarp. Nineteen trips were accomplished to collect Bryophyta, Pteridophyta and Magnoliophyta. 319 taxa, 255 on the plateau and 117 on the southern scarp were registered. The presence of distinct abiotic factors, the southern scarp being has drier than the plateau, justify the floristic difference. In spite of the low similarity level between the two sites $(12,5 \%)$, the cluster analysis grouped both with a other surveys realized in other montane forests in the northeast region. The dense forest has many species with a wide distribution in the Amazon and Atlantic regions, while in the open forest some species are more related with the thorny decidual woodland (caatinga), white others have a wide distribution in neotropical forests.
\end{abstract}

Key words - Upland forest, thorny decidual woodland, floristic, semi-arid, northeast of Brazil

\footnotetext{
${ }^{1}$ Trabalho financiado pelo projeto "Composição florística e diversidade dos brejos de altitude de Pernambuco" (Darwin Initiative/CNPq/PNE/UFRPE).

${ }^{2}$ Departamento de Biologia, UFRPE, R. Dom Manoel de Medeiros s/n., Recife, PE, Brasil. CEP 52.171-900 (rodal@truenet.com.br)

${ }^{3}$ Bolsista DTI-CNPq/PNE. Departamento de Biologia, UFRPE, R. Dom Manoel de Medeiros s/n., Recife, PE, Brasil. CEP 52171-900 (1.m.nascimento@bol.com.br)
} 


\section{Introdução}

O bioma caatinga, inserido no domínio do semi-árido, representa, segundo Heywood (1997), um importante centro de biodiversidade da América. Esse domínio ocupa uma área de $788.064 \mathrm{~km}^{2}$, equivalente a $48 \%$ da região Nordeste - sensu IBGE (Souza et al., 1994) e abriga, além da vegetação caducifólia espinhosa (VCE), a caatinga propriamente dita, outras formações vegetacionais com fisionomia e flora diferenciadas, como as florestas, e cerrados situados nos enclaves úmidos e subúmidos, totalizando $42.855,9 \mathrm{~km}^{2}$, e extensas faixas ecotonais com o cerrado e a floresta atlântica (Souza et al., 1994).

Das diferentes formações vegetacionais daquele bioma, as florestas situadas nas serras são, sem dúvida, as de maior riqueza florística (Andrade-Lima, 1973). Entre os autores que estudaram essas florestas merece destaque Dárdano de Andrade-Lima abordando a flora (Andrade-Lima, 1954; 1957; 1982), a fisionomia da vegetação (Andrade-Lima, 1961) e o perfil fitoecológico (Oliveira \& Andrade-Lima, 1948; Andrade-Lima, 1966). Recentemente, os estudos foram retomados em Pernambuco com o mapeamento dos remanescentes (Rodal et al., 1998b) e a elaboração de dois checklists (Pereira et al., 1993; Sales et al., 1998).

Em termos fisionômicos, o tipo dominante nessas serras é a floresta densa, chamada floresta serrana ou brejo de altitude, associada à floresta aberta nas escarpas médias e inferiores voltadas para a direção dos ventos, à vegetação caducifólia espinhosa (VCE) nas escarpas à sotavento da serra ou do maciço onde a serra ocorre e, eventualmente, vegetação rupícola em locais com afloramentos de rocha (Rodal et al., 1998b).

As florestas serranas pernambucanas ocorrem sempre a barlavento, nos topos e encostas superiores de algumas serras (600 a $1100 \mathrm{~m}$ ) situadas na superfície da Borborema
(Sales et al., 1998). Apesar da estreita relação entre estas florestas e condições de disponibilidade hídrica mais favoráveis que no semi-árido (Vasconcelos-Sobrinho, 1949), não existem registros pluviométricos sistemáticos nos locais onde elas ocorrem, apenas estimativas obtidas através de equações de regressão.

Ferraz et al. (1998), estudando duas formações vegetacionais do semi-árido de Pernambuco, distantes $30 \mathrm{~km}$, citaram precipitações médias anuais de $1260 \mathrm{~mm}$ em uma área de floresta serrana localizada a $1.100 \mathrm{~m}$ de altitude, contra $679 \mathrm{~mm}$ numa área da VCE a $500 \mathrm{~m}$. Em outro estudo comparativo, entre estas formações, separadas por $20 \mathrm{~km}$, Lyra (1982) observou que, apesar das duas áreas apresentarem uma diferença altitudinal de apenas $137 \mathrm{~m}$, a floresta serrana apresentou precipitação média de 948,6 mm/ano e a VCE adjacente 526,9 $\mathrm{mm}$. A autora acrescentou que no mês de setembro/81 (mês equinocial), a floresta serrana apresentou menores valores de temperatura do ar $\left(17,7^{\circ} \mathrm{C}\right)$ e maiores de umidade relativa $(82,6 \%)$, que os da $\operatorname{VCE}\left(22,6^{\circ} \mathrm{C}\right.$ e $\left.66,8 \%\right)$. Concluindo, assinalou que as florestas serranas têm menores amplitudes, tanto térmicas quanto da umidade relativa, que a VCE. Outro parâmetro climático importante nas florestas serranas é a chamada "precipitação oculta", resultado da intensa condensação noturna, especialmente nos meses mais frios (Vasconcelos-Sobrinho, 1949).

Se por um lado, a distinção fisionômica, florística e ambiental entre a floresta serrana e a VCE é clara, a transição entre esses tipos não é abrupta, sendo possível encontrar diferentes fisionomias e floras, dependendo da altitude e da exposição do relevo.

Considerando essas diferenças, este trabalho descreve a composição florística em dois pontos de uma floresta serrana situada na Reserva Biológica de Serra Negra (RBSN), estado de Pernambuco, com a finalidade avaliar as relações de similaridade entre elas e com 
outras formações do semi-árido nordestino e discutir os padrões de distribuição de algumas espécies, especialmente com base nos dados de estudos taxonômicos.

\section{Material e métodos}

Área de estudo - A RBSN situa-se no semiárido pernambucano, entre os municípios de Inajá e Floresta. Apresenta 1.100 ha $\left(8^{\circ} 35^{\prime}-8^{\circ} 38^{\prime}\right.$ S e $38^{\circ} 02^{\prime}-38^{\circ} 04^{\prime}$ W) e é a única unidade de conservação federal de floresta serrana no estado. Foi criada em 20 de setembro de 1982, de acordo com o Decreto Federal $n^{\circ} 87.591$ (IBAMA, 1989).

O relevo da RBSN é do tipo cuesta, com o topo medindo cerca $800 \mathrm{~m}$ de largura por 3.000 $\mathrm{m}$ de comprimento, orientado no sentido E/NE $\mathrm{O} / \mathrm{SO}$, com altitudes entre 800 e $1.036 \mathrm{~m}$ (Andrade, 1954). A escarpa setentrional (N/NO) apresenta um declive abrupto ao contrário da escarpa meridional (S/SE) cujo declive é suave, com altitudes variando de 550 a $800 \mathrm{~m}$ (figura 1). Trata-se de um tabuleiro relictual de uma extensa cobertura sedimentar, modelada em uma série de paleoclimas com diversos graus de umidade, que se dilatava para a direção noroeste, atingindo os estados do Ceará e Piauí, antes das sucessivas etapas de intensa erosão pelas quais passou a região Nordeste como um todo (Andrade, 1954).

A área está incluída na bacia sedimentar do Jatobá, em terrenos do Cretáceo Inferior das formações Santana e Exú, do grupo Araripe (Dantas, 1980). Os solos são arenosos amarelados e avermelhados, friáveis, argilosos, ocasionalmente caulínicos, apresentando estratificação cruzada e plano-paralela, granulometria variável, por vezes conglomeráticos, e partes com intensa silicificação (Dantas, 1980). As classes de solo citadas são: Bruno não cálcico, Areia Quartzosa Distrófica e Podzólico Vermelho - Amarelo Eutrófico, sendo as duas últimas referidas para as cotas mais elevadas da serra (IBAMA, 1989).
A precipitação e temperatura médias anuais são de $900 \mathrm{~mm}$ e $25,3^{\circ} \mathrm{C}$, respectivamente, havendo registro de temperaturas máxima e mínima absolutas de $38^{\circ} \mathrm{C}$ e $8^{\circ} \mathrm{C}$ (IBAMA, 1989). A altitude e a exposição aos ventos de sudeste, que entram pela calha do rio São Francisco, são os principais responsáveis pelo mesoclima encontrado no topo.

A floresta do topo é densa, com troncos grossos e retilíneos e emergentes de até $35 \mathrm{~m}$, que perdem as folhas no período desfavorável. $\mathrm{Na}$ escarpa meridional a floresta é aberta, as emergentes, também caducifólias, têm no máximo $20 \mathrm{~m}$ de altura e apresentam troncos que se ramificam entre três e quatro metros de altura. De um modo geral, o ambiente torna-se menos úmido, à medida que se afasta do topo em direção à base da serra, onde ocorre VCE.

Coleta e tratamento de dados - As coletas mensais de material (ervas, epífitas, lianas, trepadeiras, subarbustos, arbustos e árvores) foram realizadas no topo e na escarpa meridional, no período de 19 meses, seguindo os métodos usuais (Bridson \& Forman, 1998). As exsicatas foram processadas e depositadas no herbário PEUFR (Professor Vasconcelos-Sobrinho) da Universidade Federal Rural de Pernambuco, com duplicatas encaminhadas a diversos especialistas para confirmação ou identificação. As espécies foram organizadas por família nos sistemas de Cronquist (1981), Tryon \& Tryon (1982) e Vitt (1984), para Magnoliophyta, Pteridophyta e Bryophyta, respectivamente, incluindo informações de hábito (Font Quer, 1977). A grafia dos gêneros e a autoria das espécies foram verificadas através de Brummitt (1992) e Brummitt \& Powell (1992).

A fim de identificar com qual(is) formação(ões) vegetacional(is) ocorrentes no semi-árido nordestino a flora lenhosa do topo e da escarpa meridional teria maior similaridade, foi empregada a técnica de análise de agrupamento (Kent \& Coker,1992) para um 
conjunto de 13 listas florísticas de seis levantamentos, incluindo as duas listas da área de estudo. Foram consideradas as espécies lenhosas (árvores e arbustos), presentes no topo e na escarpa meridional, uma vez que nos demais tipos vegetacionais analisados aqueles hábitos foram incluídos. Além dessas duas listas, foram analisadas duas de floresta serrana (Ferraz et $a l ., 1998)$, uma de vegetação arbustiva perenifólia em chapada sedimentar (Rodal et al., 1998a), oito listas de vegetação caducifólia, quatro em vegetação da VCE (Rodal, 1992; Ferraz et al., 1998), três em vegetação caducifólia não espinhosa - VCNE, o chamado "carrasco" (Araújo et al., 1998) e uma na transição "carrasco-caatinga de areia" (Oliveira et al., 1997). Em seguida, foi analisada a distribuição de alguns taxa, especialmente a partir de revisões. Foi empregada a análise de agrupamentos usando o índice de similaridade de Jaccard e a técnica de aglomeração por pesos proporcionais (UPGMA) (Valentim, 2000), com auxílio do pacote FITOPAC (Shepperd, 1995).

\section{Resultados e discussão}

No total, foram encontrados 319 taxa, $85 \%$ identificados no nível de espécie e $14 \%$ no genérico, com um taxon em nível de família (tabela 1). No topo e escarpa meridional, foram coletados 255 e 117 taxa, respectivamente, com predomínio do componente lenhoso (árvores e arbustos) (figura 2). Houve proporção semelhante de espécies arbóreas em ambos, o que era de se esperar, tratando-se de duas fisionomias florestais, predomínio de epífitas na floresta densa, provavelmente em função da maior umidade; maior porcentagem de arbustos na floresta aberta, o que pode ser explicado pela maior penetração de luz, devido ao dossel ser mais aberto, bem como por se tratar de um ambiente mais seco. Apesar de próximas, o índice de similaridade (Jaccard) foi de apenas $12,5 \%$
(53 espécies comuns), o que se justifica pela presença de condições abióticas diferenciadas. Das espécies comuns, 33 foram árvores ou arbustos, especialmente das famílias Mimosaceae, Cactaceae e Caesalpiniaceae, taxa citados entre os mais freqüentes em áreas de VCE (Rodal, 1992). Entre as famílias com três ou mais espécies, nenhuma foi exclusiva da floresta aberta, enquanto Begoniaceae, Bryaceae, Dioscoreaceae, Myrtaceae, Lycopodiaceae, Orchidaceae, Polypodiaceae (comuns em florestas mais úmidas), entre outras, foram exclusivas da floresta densa.

A análise de agrupamento, realizada com base nas 310 espécies, em 13 listas de diversas formações vegetais do semi-árido (figura 3), mostra, que num primeiro nível de similaridade, ocorreram dois grupos, um, formado exclusivamente por listas de vegetação não florestal sobre chapadas sedimentares (Oliveira et al., 1997; Araújo et al., 1998; Rodal et al., 1998a) e outro reunindo as florestas serranas de Triunfo (Ferraz et al., 1998) e de Serra Negra (este trabalho) e os levantamentos da VCE (Rodal, 1992; Ferraz et al., 1998).

O resultados da análise de agrupamento indicam que as florestas serranas se unem aos levantamentos de VCE com um nível inferior a $10 \%$ de similaridade. Embora baixa, a maior similaridade destas florestas (Triunfo e Serra Negra) com a VCE se deve à presença de espécies arbóreas como: Allophylus quercifolius (Sapindaceae), Caesalpinia ferrea e Senna spectabilis var. excelsa, (Caesalpiniaceae), Coutarea hexandra (Rubiaceae), Maprounea guianensis var. guianensis (Euphorbiaceae), Parapiptadenia zehntneri (Mimosaceae), Sideroxylon obtusifolium (Sapotaceae), Tabebuia impetiginosa (Bignoniaceae) e Ziziphus joazeiro (Rhamnaceae), citadas em áreas mais úmidas da VCE nordestina (AndradeLima, 1989; Araújo et al., 1995; Prado \& Gibbs, 1993). Essa maior semelhança se justifica quando se observa que as florestas serranas estão 
Tabela 1. Famílias e espécies registradas na floresta da Reserva Biológica de Serra Negra, Floresta/ Inajá, Pernambuco, com nome, número de coletor $(\mathrm{N})$ e hábito (1- árvore; 2- arbusto; 3- subarbusto; 4- erva; 5- trepadeira; 6- liana; 7- epífita). H - hábito; T- topo e EM- escarpa meridional. Especialistas que auxiliaram na determinação de todos ou parte dos taxa estão listados após cada família.

\begin{tabular}{|c|c|c|c|c|}
\hline Divisão/Familía/Espécie & Coletor & $\mathrm{H}$ & $\mathrm{T}$ & EM \\
\hline \multicolumn{5}{|l|}{ BRYOPHYTA } \\
\hline \multicolumn{5}{|l|}{ BRYACEAE (K. Porto - UFP) } \\
\hline Brachymenium sp & D.S.Pimentel,146 & 7 & $X$ & \\
\hline Bryum cf. beyrichianum (Hornsch.) C.Muell. & J.R.R.Cantarelli,14 & 7 & $\mathrm{X}$ & \\
\hline Bryum billarderi Schwayr. & D.S.Pimentel,376 & 7 & $\mathrm{X}$ & \\
\hline \multicolumn{5}{|l|}{ PTERIDOPHYTA } \\
\hline \multicolumn{5}{|l|}{ ADIANTACEAE (P.G.Windisch - UNESP) } \\
\hline Hemionitis tomentosa (Lam.) Raddi & D.S.Pimentel,142 & 7 & $\mathrm{X}$ & \\
\hline \multicolumn{5}{|l|}{ ASPLENIACEAE (P.G.Windisch - UNESP) } \\
\hline Asplenium punilum $\mathrm{Sw}$. & A.P.S.Gomes,56 & 7 & $\mathrm{X}$ & \\
\hline \multicolumn{5}{|l|}{ LYCOPODIACEAE(I.C.L.Barros - UFP) } \\
\hline Huperzia chiristii (Silveira) Holub. & D.S.Pimentel,s/n2 & 7 & $X$ & \\
\hline Huperzia flexibilis (Fée) B.Øllg. & D.S.Pimentel,s/n1 & 7 & $X$ & \\
\hline \multicolumn{5}{|c|}{ POLYPODIACEAE (I.C.L.Barros - UFP; P.G. Windisch - UNESP; J.TMickel -NY) } \\
\hline Microgramma squamulosa (Kef.) de la Sota & D.S.Pimentel,140 & 7 & $X$ & \\
\hline Microgramma crispata (Fée) de la Sota & M.J.N.Rodal, 478 & 7 & $\mathrm{X}$ & \\
\hline Pleopeltis angusta Willd. & M.F.Sales, 640 & 7 & $\mathrm{X}$ & \\
\hline Pleopeltis astrolepis Liebm. & D.S.Pimentel,362 & 7 & $\mathrm{X}$ & \\
\hline Polypodium aureum $\mathrm{L}$. & A.Laurênio,167 & 7 & $\mathrm{X}$ & \\
\hline Polypodium brasiliense Poir. & M.J.N.Rodal, 654 & 7 & $\mathrm{X}$ & \\
\hline Polypodium hirsutissimum Raddi & D.S.Pimentel,357 & 7 & $\mathrm{X}$ & \\
\hline \multicolumn{5}{|c|}{ MAGNOLIOPHYTA } \\
\hline \multicolumn{5}{|c|}{ ACANTHACEAE (M.Wasshausen - NY; M.R.C.Sales - PEUFR) } \\
\hline Dicliptera ciliaris A.Juss. & M.F.Sales,339 & 4 & $X$ & \\
\hline Justicia cf. thumbergioides (Lindau) Leonard & A.P.S.Gomes,554 & 4 & $X$ & \\
\hline Justicia strobilacea (Nees) Lindau & A.P.S.Gomes, 3 & 3 & $\mathrm{X}$ & \\
\hline Ruellia asperula (Nees) Lindau & A.Laurênio,156 & 2 & & $\mathrm{X}$ \\
\hline Ruellia bahiensis (Nees) Morong & M.Oliveira,323 & 2 & & $\mathrm{X}$ \\
\hline Ruellia cf. geminiflora Humb., Bonpl. \& Kunth & M.J.N.Rodal,349 & 5 & & $\mathrm{X}$ \\
\hline \multicolumn{5}{|l|}{ ALSTROEMERIACEAE(P.Wilken - K) } \\
\hline Bomarea edulis Mirb. & M.F.Sales, 642 & 5 & $\mathrm{X}$ & \\
\hline \multicolumn{5}{|l|}{ AMARANTHACEAE } \\
\hline Alternanthera brasiliana (L.) Kuntze & M.J.N.Rodal,484 & 2 & $X$ & \\
\hline Gomphrena vaga Mart. & M.J.N.Rodal,626 & 3 & $\mathrm{X}$ & \\
\hline \multicolumn{5}{|l|}{ AMARYLLIDACEAE } \\
\hline Hippeastrum $\mathrm{sp}$ & A.Laurênio,269 & 4 & $\mathrm{X}$ & \\
\hline \multicolumn{5}{|l|}{ ANACARDIACEAE } \\
\hline Schinopsis brasiliensis Engl. & A.Laurênio,98 & 1 & $\mathrm{X}$ & \\
\hline \multicolumn{5}{|l|}{ ANNONACEAE } \\
\hline Rollinia leptopetala (R.E.Fries) Safford & E.Inácio,256 & 2 & $X$ & \\
\hline \multicolumn{5}{|l|}{ APOCYNACEAE(M.F.Sales - PEUFR) } \\
\hline Aspidosperma cf. illustre (Vell.) Kuhlm. \& Piraja & L.M.Nascimento,41 & 1 & $X$ & \\
\hline Aspidosperma pyrifolium Mart. & A.P.S.Gomes, 211 & 1 & $X$ & \\
\hline
\end{tabular}


Tabela 1. (continuação).

\begin{tabular}{|c|c|c|c|c|}
\hline Divisão/Familía/Espécie & Coletor & $\mathrm{H}$ & $\mathrm{T}$ & $\mathrm{EM}$ \\
\hline Aspidosperma sp1 & M.J.N.Rodal,611 & 1 & $X$ & \\
\hline Aspidosperma $\mathrm{sp} 2$ & M.J.N.Rodal,705 & 1 & $X$ & \\
\hline \multicolumn{5}{|l|}{ ARACEAE (S.J.Mayo - K) } \\
\hline Anthurium cf. affine Schott & A.Laurênio,157 & 4 & $\mathrm{X}$ & \\
\hline Anthurium scandens (Aubl.) Engl. & A.Laurênio, 153 & 7 & $X$ & \\
\hline \multicolumn{5}{|l|}{ ASCLEPIADACEAE (D.Goyder - K; J.Fontella - HB ) } \\
\hline Ditassa glaziovii Fourn. & M.F.Sales,535 & 5 & $X$ & \\
\hline Ditassa oxyphylla Turcz. & M.J.N.Rodal,487 & 5 & $X$ & \\
\hline Schubertia $\mathrm{sp}$ & M.F.Sales,634 & 5 & $\mathrm{X}$ & \\
\hline \multicolumn{5}{|c|}{ ASTERACEAE (J.A.Bastos - PEUFR; N.Hind - K.; H.Robinson - NY) } \\
\hline Baccharis serrulata (Lam.) Pers. & M.C.Tschá,384 & 2 & & $\mathrm{X}$ \\
\hline Baccharis sp & D.S.Pimentel,379 & 3 & & $\mathrm{X}$ \\
\hline Conocliniopsis prasiifolia (DC.) R.M.King \& H.Rob. & A.Laurênio,77 & 3 & & $\mathrm{X}$ \\
\hline Conyza bonariensis (L.) Cronquist & L.S.Figueirêdo, 10 & 4 & & $\mathrm{X}$ \\
\hline Eupatorium ballataefolium Humb., Bonpl. \& Kunth & D.S.Pimentel,368 & 4 & & $\mathrm{X}$ \\
\hline Eupatorium sp & D.S.Pimentel,372 & 4 & & $\mathrm{X}$ \\
\hline Koanophyllum conglobatum (DC.) R.M.King \& H.Rob. & L.S.Figueirêdo,6 & 2 & $\mathrm{X}$ & \\
\hline Mikania cordifolia (L.f.) Willd. & A.Laurênio,164 & 5 & $\mathrm{X}$ & \\
\hline Trixis divaricata (Kunth) Spreng. & M.C.Tschá,18 & 3 & $\mathrm{X}$ & \\
\hline Verbesina macrophylla (Cass.) S.F.Blake & M.J.N.Rodal, 606 & 2 & $\mathrm{X}$ & \\
\hline Vernonia chalybaea Mart. ex DC. & D.S.Pimentel,371 & 3 & & $\mathrm{X}$ \\
\hline Wedellia alagoensis Baker & M.J.N.Rodal,356 & 3 & $\mathrm{X}$ & \\
\hline Wedellia hookeriana Gardner & E.Inácio, 1 & 2 & $X$ & \\
\hline Wedellia villosa Gardner & L.M.Nascimento,70 & 3 & & $\mathrm{X}$ \\
\hline \multicolumn{5}{|l|}{ BEGONIACEAE (E.Jacques - CEUL) } \\
\hline Begonia cf. grisea A.DC. & M.F.Sales,551 & 4 & $X$ & \\
\hline Begonia petassifolia Brade & M.F.Sales, 647 & 4 & $\mathrm{X}$ & \\
\hline Begonia reniformis Dryand. & M.J.N.Rodal,623 & 4 & $\mathrm{X}$ & \\
\hline Begonia sp & D.S.Pimentel,331 & 4 & $X$ & \\
\hline \multicolumn{5}{|l|}{ BIGNONIACEAE (C.Sothers - K) } \\
\hline Amphilophius sp & E.Inácio, 85 & 5 & $X$ & \\
\hline Anemopaegma laeve DC. & M.F.A.Lucena, 15 & 6 & $\mathrm{X}$ & \\
\hline Arrabidaea $\mathrm{sp}$ & M.F.Sales, 630 & 6 & & $\mathrm{X}$ \\
\hline Clytostoma binatum (Thumb.) Sandw. & M.J.N.Rodal,637 & 5 & $\mathrm{X}$ & \\
\hline Memora cristicalyx A.Gentry & A.Laurênio,180 & 5 & & $X$ \\
\hline Memora sp & M.C.Tschá,123 & 5 & & $\mathrm{X}$ \\
\hline Parabignonia sp & M.F.Sales,344 & 5 & $X$ & \\
\hline Pyrostegia venusta (Ker Gawl.) Miers & A.P.S.Gomes, 120 & 5 & $\mathrm{X}$ & \\
\hline Tabebuia impetiginosa (Mart.ex DC.) Standl. & A.P.S.Gomes, 214 & 1 & $X$ & \\
\hline Tabebuia serratifolia (Vahl) G.Nicholson & M.J.N.Rodal,720 & 1 & $X$ & \\
\hline \multicolumn{5}{|l|}{ BORAGINACEAE (S.F.Smith - NY) } \\
\hline Cordia curassavica (Jacq.) Roem. \& Schult. & K.Andrade,5 & 2 & $X$ & $\mathrm{X}$ \\
\hline Cordia leucomalloides Taroda & M.J.N.Rodal,481 & 2 & $\mathrm{X}$ & $\mathrm{X}$ \\
\hline Cordia sp1 & E.Inácio,103 & 2 & $\mathrm{X}$ & \\
\hline Cordia $\mathrm{sp} 2$ & E.Inácio,260 & 2 & $X$ & \\
\hline Heliotropium angiospermum Murr. & L.F.Silva,28 & 4 & $\mathrm{X}$ & \\
\hline
\end{tabular}


Tabela 1. (continuação).

\begin{tabular}{|c|c|c|c|c|}
\hline Divisão/Familía/Espécie & Coletor & $\mathrm{H}$ & $\mathrm{T}$ & $\mathrm{EM}$ \\
\hline Heliotropium tiaridioides Cham. & F.M.O.Villarouco, 17 & 4 & & $\mathrm{X}$ \\
\hline Tournefortia $\mathrm{sp}$ & M.J.N.Rodal,346 & 4 & & $\mathrm{X}$ \\
\hline \multicolumn{5}{|c|}{ BROMELIACEAE (G.M.Souza - PFPB; M.G.L.Wanderley - SP) } \\
\hline Aechmea tomentosa $\mathrm{Mez}$ & M.F.Sales, 560 & 7 & $\mathrm{X}$ & \\
\hline Bilbergia porteana Brongn. & G.M.Sousa,68 & 7 & $\mathrm{X}$ & \\
\hline Portea leptantha Harms & G.M.Sousa,69 & 7 & $\mathrm{X}$ & \\
\hline Tillandsia gardneri Lindl. & G.M.Sousa,65 & 7 & $\mathrm{X}$ & $\mathrm{X}$ \\
\hline Tillandsia juncea (Ruíz \& Pav.) Poir. & D.S.Pimentel,349 & 7 & $\mathrm{X}$ & \\
\hline Tillandsia polystachia (L.) L. & G.M.Sousa,71 & 7 & $\mathrm{X}$ & \\
\hline Tillandsia recurvata $\mathrm{L}$. & D.S.Pimentel,335 & 7 & $\mathrm{X}$ & \\
\hline Tillandsia tenuifolia $\mathrm{L}$. & G.M.Sousa,99 & 7 & $\mathrm{X}$ & \\
\hline Tillandsia usneoides (L.) L. & G.M.Sousa,66 & 7 & $\mathrm{X}$ & \\
\hline Bromeliaceae & D.S.Pimentel,337 & 7 & $\mathrm{X}$ & \\
\hline \multicolumn{5}{|l|}{ CACTACEAE(D.Zappi - K) } \\
\hline Cereus jamacaru DC. & M.J.N.Rodal, 718 & 2 & $\mathrm{X}$ & $\mathrm{X}$ \\
\hline Harrisia adscendens (Gürke) Britton \& Rose & A.Laurênio,265 & 2 & & $\mathrm{X}$ \\
\hline Melocactus sp & A.Laurênio,199 & 4 & & $\mathrm{X}$ \\
\hline Opuntia brasiliensis (Willd.) Haw. & M.F.Sales,564 & 1 & $\mathrm{X}$ & $\mathrm{X}$ \\
\hline Pilosocereus catingicola subsp. salvadorensis & $\begin{array}{l}\text { (Werderm.) Zappi } \\
\text { A.Laurênio,196 }\end{array}$ & 2 & $\mathrm{X}$ & $\mathrm{X}$ \\
\hline Rhipsalis baccifera (J.S.Muell.) Stearn & A.Laurênio, 159 & 7 & $\mathrm{X}$ & $\mathrm{X}$ \\
\hline Rhipsalis sp & A.P.S.Gomes, 143 & 7 & $\mathrm{X}$ & \\
\hline \multicolumn{5}{|l|}{ CAESALPINIACEAE (G.P.Lewis- K ; R.Barneby - NY) } \\
\hline Caesalpinia ferrea Mart. ex Tul. & A.Laurênio,299 & 1 & $\mathrm{X}$ & \\
\hline Caesalpinia pyramidalis Tul. & A.Laurênio,191 & 1 & & $\mathrm{X}$ \\
\hline Senna macranthera (Vogel) H.S.Irwin \& Barneby & E.Inácio,98 & 2 & $\mathrm{X}$ & $\mathrm{X}$ \\
\hline $\begin{array}{l}\text { Senna macranthera var. micans (Nees) H.S. } \\
\text { Irwin \& Barneby }\end{array}$ & E.Ferraz,262 & 1 & $X$ & $X$ \\
\hline $\begin{array}{l}\text { Senna macranthera var. pudibunda (Nees) H.S. } \\
\text { Irwin \& Barneby }\end{array}$ & A.Laurênio,72 & 1 & $\mathrm{X}$ & $\mathrm{X}$ \\
\hline Senna pendula var. dolichandra H.S.Irwin \& Barneby & C.S.Zickel,14 & 2 & & $\mathrm{X}$ \\
\hline Senna spectabilis (DC.) H.S.Irwin \& Barneby & E.Inácio, 168 & 2 & $\mathrm{X}$ & $\mathrm{X}$ \\
\hline $\begin{array}{l}\text { Senna spectabilis var. excelsa (Schrad.) H.S. } \\
\text { Irwin \& Barneby }\end{array}$ & E.Menezes,39 & 2 & $\mathrm{X}$ & $\mathrm{X}$ \\
\hline $\begin{array}{l}\text { Senna trachypus (Benth.) H.S.Irwin \& Barneby } \\
\text { CAPPARACEAE (M.B.Costa e Silva - PEUFR) }\end{array}$ & M.C.Tschá,261 & 2 & & $\mathrm{X}$ \\
\hline Capparis flexuosa (L.) L. & L.M.Nascimento,29 & 2 & $X$ & $\mathrm{X}$ \\
\hline Capparis jacobinae Moric. ex Eichler & L.M.Nascimento, 15 & 2 & $\mathrm{X}$ & $\mathrm{X}$ \\
\hline $\begin{array}{l}\text { Capparis yco Mart. \& Eichler } \\
\text { CELASTRACEAE }\end{array}$ & L.M.Nascimento, 14 & 2 & $\mathrm{X}$ & $\mathrm{X}$ \\
\hline Maytenus aff. evonymoides Reissek & M.C.Tschá,233 & 1 & $\mathrm{X}$ & \\
\hline Maytenus obtusifolia Mart. & L.M.Nascimento, 24 & 1 & $\mathrm{X}$ & \\
\hline $\begin{array}{l}\text { Maytenus rigida } \text { Mart. } \\
\text { CLUSIACEAE }\end{array}$ & M.F.Sales,313 & 1 & $\mathrm{X}$ & $\mathrm{X}$ \\
\hline $\begin{array}{l}\text { Garcinia gardneriana (Planch. \& Triana) Zappi } \\
\text { COMBRETACEAE }\end{array}$ & L.M.Nascimento, 40 & 1 & $\mathrm{X}$ & \\
\hline Buchenavia capitata (Vahl) Eichler & L.M.Nascimento,42 & 1 & $\mathrm{X}$ & \\
\hline
\end{tabular}


Tabela 1. (continuação).

\begin{tabular}{|c|c|c|c|c|}
\hline Divisão/Familía/Espécie & Coletor & $\mathrm{H}$ & $\mathrm{T}$ & $\mathrm{EM}$ \\
\hline $\begin{array}{l}\text { Terminalia brasiliensis Cambess. } \\
\text { COMMELINACEAE (R. Barreto - UFP) }\end{array}$ & M.J.N.Rodal,569 & 1 & $X$ & \\
\hline Aneilema brasiliensis C.B.Clarke & M.F.Sales,307 & 4 & & $X$ \\
\hline Callisia monandra (Sw.) Schult.f. & M.F.Sales,306 & 4 & $\mathrm{X}$ & \\
\hline Callisia repens $\mathrm{L}$. & M.F.Sales,323 & 4 & $\mathrm{X}$ & $X$ \\
\hline Commelina erecta $\mathrm{L}$. & L.F.Silva,27 & 4 & $\mathrm{X}$ & \\
\hline $\begin{array}{l}\text { Dichorisandra hexandra (Aubl.) Standl. } \\
\text { CONVOLVULACEAE }\end{array}$ & M.F.Sales, 613 & 4 & $X$ & $X$ \\
\hline Aniseia $s p$ & M.F.Sales, 618 & 5 & & $X$ \\
\hline Evolvulus elegans Moric. & M.C.Tschá,159 & 3 & $\mathrm{X}$ & \\
\hline Evolvulus latifolius Ker Gawl. & M.J.N.Rodal,354 & 3 & $\mathrm{X}$ & \\
\hline Jacquemontia ferruginea Choisy & D.C.Silva,78 & 4 & $\mathrm{X}$ & \\
\hline $\begin{array}{l}\text { Jacquemontia nodiflora (Desv.) G. Don } \\
\text { CUCURBITACEAE (C.Sothers - K) }\end{array}$ & M.F.Sales, 305 & 5 & $\mathrm{X}$ & \\
\hline Cayaponia racemosa (Mill.) Cogn. & M.J.N.Rodal,666 & 5 & $X$ & \\
\hline $\begin{array}{l}\text { Momordica charantia L. } \\
\text { DIOSCOREACEAE (G.Pedralli - HXBH; P.Wilken - K) }\end{array}$ & F.M.O.Villarouco, 19 & 5 & & $X$ \\
\hline Dioscorea dodecaneura Vell. & M.F.Sales, 614 & 5 & $X$ & \\
\hline Dioscorea monadelpha (Kunth) Griseb. & M.F.Sales,662 & 5 & $X$ & \\
\hline Dioscorea piperifolia Humb. \& Bonpl. ex Willd. & M.C.Tschá,218 & 5 & $\mathrm{X}$ & \\
\hline $\begin{array}{l}\text { Dioscorea polygonoides Humb. \& Bonpl. ex Willd. } \\
\text { EBENACEAE }\end{array}$ & M.Oliveira,318 & 5 & $X$ & \\
\hline Diospyros cf. brasiliensis Mart. & M.C.Tschá,234 & 1 & $\mathrm{X}$ & \\
\hline ERYTHROXYLACEAE (S.F.Smith - K; M.I.B.Loyola - & EUFR) & & & \\
\hline Erythroxylum distortum Mart. & M.F.Sales,608 & 2 & $\mathrm{X}$ & $\mathrm{X}$ \\
\hline Erythroxylum macrochaetum Miguel & L.M.Nascimento, 12 & 2 & $\mathrm{X}$ & \\
\hline Erythroxylum sp & M.C.Tschá,14 & 2 & $\mathrm{X}$ & \\
\hline EUPHORBIACEAE (G.L.Webster-DAV; M.F.A.Luce & - PEUFR) & & & \\
\hline Acalypha pruriens Nees \& Mart. & M.F.Sales,293 & 3 & $\mathrm{X}$ & \\
\hline Croton conduplicatus Kunth & A.B.Marcon, 11 & 3 & $\mathrm{X}$ & $\mathrm{X}$ \\
\hline Croton macrocalyx Mart. ex Baill. & M.F.Sales, 624 & 2 & $X$ & $\mathrm{X}$ \\
\hline Croton micans (Sw.) Müll.Arg. & L.F.Silva,261 & 2 & $\mathrm{X}$ & $\mathrm{X}$ \\
\hline Croton moritibensis Baill. & K.Andrade, 4 & 2 & $X$ & $\mathrm{X}$ \\
\hline Croton pulegioides Baill. & M.F.Sales, 653 & 3 & $X$ & $\mathrm{X}$ \\
\hline Croton rhamnifolius (Baill.) Müll.Arg. & M.J.Hora,113 & 3 & & $\mathrm{X}$ \\
\hline Dalechampia cf. brasiliensis Lam. & D.C.Silva,94 & 5 & $X$ & $\mathrm{X}$ \\
\hline Dalechampia olfersiana Müll.Arg & M.J.N.Rodal,456 & 5 & $X$ & $\mathrm{X}$ \\
\hline Jatropha molissima (Pohl) Baill. & M.J.N.Rodal,688 & 2 & & $\mathrm{X}$ \\
\hline Maprounea guianensis Aubl. var. guianensis & L.M.Nascimento, 68 & 1 & $X$ & \\
\hline Phyllanthus cf. amarus K.Schum. & D.C.Silva,9 & 4 & $X$ & \\
\hline Sebastiania brasiliensis Spreng. & M.F.Sales, 616 & 1 & $\mathrm{X}$ & \\
\hline Tragia cf. alienata (Didr.) Múlgura. \& M.M.Gut. & M.F.A.Lucena,136 & 5 & $X$ & \\
\hline $\begin{array}{l}\text { Tragia volubilis L. } \\
\text { FABACEAE(G.P.Lewis - K; L.P. de Queiroz - UEFS) }\end{array}$ & A.Laurênio,7 & 5 & $\mathrm{X}$ & \\
\hline Bocoa mollis (Benth.) R.S.Cowan & M.J.N.Rodal,706 & 2 & & $\mathrm{X}$ \\
\hline
\end{tabular}


Tabela 1. (continuação).

\begin{tabular}{|c|c|c|c|c|}
\hline Divisão/Familía/Espécie & Coletor & $\mathrm{H}$ & $\mathrm{T}$ & $\mathrm{EM}$ \\
\hline Chaetocalyx longiflora A.Gray & M.F.Sales,322 & 2 & $\mathrm{X}$ & \\
\hline Chaetocalyx scandens var. pubescens (DC.) Rudd & E.Ferraz,272 & 2 & & $\mathrm{X}$ \\
\hline Chaetocalyx sp & A.P.S.Gomes, 57 & 2 & & $\mathrm{X}$ \\
\hline Crotalaria vitellina Ker Gawl. & A.P.S.Gomes, 149 & 2 & $X$ & $\mathrm{X}$ \\
\hline Dalbergia catingicola Harms & A.Laurênio, 170 & 2 & & $X$ \\
\hline Dalbergia cearensis Ducke & A.P.S.Gomes, 7 & 1 & & $\mathrm{X}$ \\
\hline Dioclea grandiflora Mart. ex Benth. & E.Inácio, 169 & 5 & & $X$ \\
\hline Indigofera suffruticosa Mill. & M.C.Tschá,118 & 3 & $\mathrm{X}$ & $\mathrm{X}$ \\
\hline Lonchocarpus sp1 & A.Laurênio,264 & 1 & & $\mathrm{X}$ \\
\hline Lonchocarpus sp2 & A.Laurênio,181 & 1 & & $\mathrm{X}$ \\
\hline Macroptilium sp & A.P.S.Gomes, 123 & 5 & & $X$ \\
\hline Periandra coccinea (Schrad.) Benth. & A.Laurênio,5 & 5 & $\mathrm{X}$ & $\mathrm{X}$ \\
\hline Periandra mediterranea (Vell.) Conc. Taubert & M.F.Sales,326 & 5 & $\mathrm{X}$ & \\
\hline Platymiscium floribundum Vogel & M.J.N.Rodal,684 & 1 & $\mathrm{X}$ & \\
\hline Poiretia punctata (Willd.) Desv. ex Rudd & A.Laurênio,106 & 5 & $\mathrm{X}$ & $\mathrm{X}$ \\
\hline $\begin{array}{l}\text { Vigna candida (Vell.) Maréchal, Mascherpa \& Stainier } \\
\text { LAMIACEAE }\end{array}$ & M.J.N.Rodal,351 & 5 & $\mathrm{X}$ & $\mathrm{X}$ \\
\hline $\begin{array}{l}\text { Hyptis pectinata }(\text { L.) Poir. } \\
\text { LAURACEAE (R.Roxana - UFP) }\end{array}$ & E.Inácio,263 & 3 & $\mathrm{X}$ & \\
\hline $\begin{array}{l}\text { Ocotea duckei Vattimo-Gil } \\
\text { LORANTHACEAE (B.Stannard - K) }\end{array}$ & M.F.Sales,558 & 1 & $\mathrm{X}$ & \\
\hline $\begin{array}{l}\text { Struthanthus polyrrhizus Mart. } \\
\text { LYTHRACEAE(T.B.Cavalcanti-CEN) }\end{array}$ & M.F.Sales,665 & 7 & $\mathrm{X}$ & \\
\hline Cuphea racemosa (L.f.) Spreng. & M.J.N.Rodal,620 & 4 & $\mathrm{X}$ & \\
\hline MALPIGHIACEAE (W.R.Anderson - MICH; C.Sothers & $-\mathrm{K})$ & & & \\
\hline Banisteriopsis platypoda (Griseb.) Cuatrec. & M.J.N.Rodal,681 & 1 & $\mathrm{X}$ & \\
\hline Banisteriopsis sp1 & M.J.N.Rodal,662 & 1 & $\mathrm{X}$ & \\
\hline Banisteriopsis sp2 & M.J.N.Rodal,723 & 1 & $\mathrm{X}$ & \\
\hline Barnebya harleyi W.R.Anderson \& B.Gates & M.J.N.Rodal,818 & 1 & & $\mathrm{X}$ \\
\hline Bunchosia pernambucana W.R.Anderson - Typus & E.Menezes, 33 & 1 & $\mathrm{X}$ & \\
\hline Heteropterys coleoptera A.Juss. & M.F.Sales, 655 & 5 & $\mathrm{X}$ & \\
\hline Heteropterys perplexa W.R.Anderson & M.J.N.Rodal,572 & 5 & $\mathrm{X}$ & \\
\hline Heteropterys $\mathrm{sp} 1$ & A.P.S.Gomes, 220 & 5 & $\mathrm{X}$ & \\
\hline Heteropterys $\mathrm{sp} 2$ & M.C.Tschá,130 & 5 & $\mathrm{X}$ & \\
\hline Mascagnia chlorocarpa (A.Juss.) Griseb. & D.C.Silva,95 & 5 & $\mathrm{X}$ & $\mathrm{X}$ \\
\hline Ptilochaeta bahiensis Turcz. & A.Laurênio, 257 & 2 & $\mathrm{X}$ & $\mathrm{X}$ \\
\hline Thryallis longifolia Mart. & M.F.Sales, 660 & 2 & $\mathrm{X}$ & \\
\hline MALVACEAE (A.Krapovickas - G; G.S.Baracho - UFP; & P.A.Fryxell - TEX) & & & \\
\hline Gaya canescens Humb., Bonpl. \& Kunth & E.Menezes, 41 & 4 & & $\mathrm{X}$ \\
\hline Herissantia crispa $($ L.) Brizicky & E.Inácio, 8 & 2 & $\mathrm{X}$ & \\
\hline Herissantia tiubae (K.Schum.) Brizicky & L.F.Silva,22 & 3 & & $\mathrm{X}$ \\
\hline Malvastrum coromandelianum (L.) Garcke. & K.Andrade, 10 & 3 & $\mathrm{X}$ & \\
\hline Pavonia glazioviana Gürke & M.F.Sales,670 & 4 & & $\mathrm{X}$ \\
\hline Sida galheirensis Ulbr. & A.B.Marcon, 16 & 2 & & $\mathrm{X}$ \\
\hline Sidastrum multiflorum (Jacq.) Fryxell & L.F.Silva,26 & 3 & $X$ & $X$ \\
\hline
\end{tabular}


Tabela 1. (continuação).

\begin{tabular}{|c|c|c|c|c|}
\hline Divisão/Familía/Espécie & Coletor & $\mathrm{H}$ & $\mathrm{T}$ & $\mathrm{EM}$ \\
\hline $\begin{array}{l}\text { Sidastrum paniculatum (L.) Fryxell } \\
\text { MARANTACEAE (K.N.Yoshida Arns - UFP) }\end{array}$ & A.P.S.Gomes, 151 & 3 & $\mathrm{X}$ & \\
\hline $\begin{array}{l}\text { Ctenanthe pickeliana K.Y.Arns \& S.Mayo } \\
\text { MELASTOMATACEAE }\end{array}$ & L.M.Nascimento,63 & 3 & $\mathrm{X}$ & \\
\hline $\begin{array}{l}\text { Miconia } \mathrm{sp} \\
\text { MELIACEAE }\end{array}$ & M.J.N.Rodal, 672 & 1 & $X$ & \\
\hline $\begin{array}{l}\text { Trichilia emarginata (Turcz.) C.DC. } \\
\text { MENISPERMACEAE (R.Barneby - NY) }\end{array}$ & M.J.N.Rodal,622 & 1 & $X$ & \\
\hline Cissampelos pareira $\mathrm{L}$. & M.J.N.Rodal,489 & 5 & $X$ & \\
\hline \multicolumn{5}{|c|}{ MIMOSACEAE (G.P.Lewis - K; L.Rico - K; R.Barneby - NY) } \\
\hline Acacia bahiensis Benth. & M.J.N.Rodal,615 & 2 & & $\mathrm{X}$ \\
\hline Acacia riparia Kunth & A.Laurênio,102 & 1 & $\mathrm{X}$ & $\mathrm{X}$ \\
\hline Acacia tenuifolia (L.) Willd. & K.Andrade, 12 & 2 & $\mathrm{X}$ & $\mathrm{X}$ \\
\hline Acacia $\mathrm{sp} 1$ & M.F.Sales, 625 & 1 & $\mathrm{X}$ & $\mathrm{X}$ \\
\hline Acacia $\mathrm{sp} 2$ & M.J.N.Rodal,574 & 1 & $\mathrm{X}$ & $\mathrm{X}$ \\
\hline Albizia polycephala (Benth.) Killip & L.M.Nascimento,31 & 1 & $X$ & \\
\hline Chloroleucon foliolosum (Benth.) G.P.Lewis & A.P.S.Gomes, 140 & 1 & & $\mathrm{X}$ \\
\hline Inga thibaudiana DC. & M.J.N.Rodal,568 & 1 & $X$ & \\
\hline Mimosa acutistipula (Mart.) Benth. & A.P.S.Gomes, 141 & 2 & & $\mathrm{X}$ \\
\hline Mimosa acutistipula (Mart.) Benth. var. acutistipula & A.P.S.Gomes, 50 & 2 & & $\mathrm{X}$ \\
\hline Mimosa arenosa (Willd.) Poir. var. arenosa & A.P.S.Gomes, 30 & 2 & & $\mathrm{X}$ \\
\hline Mimosa ophthalmocentra Mart. ex Benth. & A.P.S.Gomes, 44 & 2 & & $\mathrm{X}$ \\
\hline Mimosa sensitiva $\mathrm{L}$. & A.P.S.Gomes,34 & 2 & & $\mathrm{X}$ \\
\hline Mimosa sensitiva L. var. sensitiva & M.Oliveira,325 & 2 & & $\mathrm{X}$ \\
\hline $\begin{array}{l}\text { Parapiptadenia zehntneri (Harms.) } \\
\text { M.P.Lima \& H.C.Lima }\end{array}$ & A.P.S.Gomes, 148 & 1 & $\mathrm{X}$ & $\mathrm{X}$ \\
\hline Piptadenia stipulacea (Benth.) Ducke & A.P.S.Gomes, 137 & 1 & $\mathrm{X}$ & $\mathrm{X}$ \\
\hline Pithecellobium diversifolium Benth. & A.Laurênio,71 & 1 & & $\mathrm{X}$ \\
\hline $\begin{array}{l}\text { Plathymenia foliolosa Benth. } \\
\text { MORACEAE }\end{array}$ & M.F.Sales, 622 & 1 & & $\mathrm{X}$ \\
\hline Ficus sp1 & M.F.Sales,649 & 1 & $\mathrm{X}$ & \\
\hline Ficus sp2 & M.C.Tschá,113 & 1 & $\mathrm{X}$ & \\
\hline MYRSINACEAE & & & & \\
\hline $\begin{array}{l}\text { Myrsine guianensis (Aubl.) Kuntze } \\
\text { MYRTACEAE(G.M.Barroso-RB) }\end{array}$ & M.J.N.Rodal,624 & 1 & $X$ & \\
\hline Calyptranthes dardanoi Mattos & L.F.Silva,265 & 1 & $X$ & \\
\hline Calyptranthes multiflora Poepp. ex Baker & L.M.Nascimento,69 & 1 & $\mathrm{X}$ & \\
\hline Eugenia biflora $(\mathrm{L}$.$) DC.$ & K.Andrade, 14 & 1 & $X$ & \\
\hline Eugenia hirta $\mathrm{O}$. Berg & L.M.Nascimento,51 & 1 & $X$ & \\
\hline Eugenia lambertiana var. hispidula McVaugh & M.J.N.Rodal,722 & 1 & $\mathrm{X}$ & $\mathrm{X}$ \\
\hline Eugenia punicifolia (Humb., Bonpl. \& Kunth) DC. & M.F.Sales, 635 & 1 & $\mathrm{X}$ & \\
\hline Myrcia aff. bullata O.Berg & L.M.Nascimento,66 & 1 & $X$ & \\
\hline Myrcia caesia O.Berg & L.M.Nascimento, 20 & 1 & $\mathrm{X}$ & \\
\hline Myrcia fallax (Rich.) DC. & M.J.N.Rodal,560 & 1 & $\mathrm{X}$ & \\
\hline Myrcia multiflora (Lam.) DC. & L.M.Nascimento, 16 & 1 & $X$ & $\mathrm{X}$ \\
\hline Myrciaria floribunda (West ex Willd.) O.Berg & L.M.Nascimento,62 & 1 & $X$ & \\
\hline
\end{tabular}


Tabela 1. (continuação).

\begin{tabular}{|c|c|c|c|c|}
\hline Divisão/Familía/Espécie & Coletor & $\mathrm{H}$ & $\mathrm{T}$ & $\mathrm{EM}$ \\
\hline $\begin{array}{l}\text { Psidium personii } \mathrm{McV} \text { Vaugh } \\
\text { NYCTAGINACEAE(A.Furlan - HRCB) }\end{array}$ & M.J.N.Rodal,644 & 1 & $\mathrm{X}$ & \\
\hline Guapira laxa (Netto) Furlan & M.J.N.Rodal,707 & 1 & $\mathrm{X}$ & $X$ \\
\hline $\begin{array}{l}\text { Guapira opposita (Vell.) Reitz } \\
\text { OLACACEAE(J.Kallunki - NY) }\end{array}$ & L.M.Nascimento,59 & 1 & $\mathrm{X}$ & \\
\hline Schoepfia brasiliensis A.DC. & M.F.Sales, 645 & 1 & $\mathrm{X}$ & \\
\hline \multicolumn{5}{|l|}{ ORCHIDACEAE (L.P.Félix - EAN; S.Thomas - K) } \\
\hline Amblostoma tridactylum (Lindl.) Rchb.f & J.R.R.Cantarelli,11 & 7 & $\mathrm{X}$ & \\
\hline Campylocentrum crassirhizum Hoehne & M.F.Sales,320 & 7 & $\mathrm{X}$ & \\
\hline Campylocentrum neglectum (Rchb.f \& Wärn) Cogn. & D.S.Pimentel,91 & 7 & $\mathrm{X}$ & \\
\hline Cattleya labiata Lindl. & D.S.Pimentel,65 & 7 & $\mathrm{X}$ & \\
\hline Cranichis cf. glabricaulis Hoehne & J.Urbano,1 & 7 & $\mathrm{X}$ & \\
\hline Cranichis scripta Kraenzl. & E.Inácio,90 & 4 & $\mathrm{X}$ & \\
\hline Cyclopogon elatus (Sw.) Schltr. & J.Urbano,3 & 7 & $\mathrm{X}$ & \\
\hline Epidendrum ramosum Jacq. & L.M.Nascimento,79 & 7 & $\mathrm{X}$ & \\
\hline Habenaria glaucophylla Barb.Rodr. & J.Urbano,2 & 4 & $\mathrm{X}$ & \\
\hline Lanium avicula (Lindl.) Benth. & L.M.Nascimento,s/n & 7 & $\mathrm{X}$ & \\
\hline Oncidium barbatum Lindl. & M.J.N.Rodal, 625 & 7 & $\mathrm{X}$ & \\
\hline Oncidium gravesianum Rolfe & D.S.Pimentel,111 & 7 & $\mathrm{X}$ & \\
\hline Pleurothallis rubens Lindl. & D.S.Pimentel,155 & 7 & $\mathrm{X}$ & \\
\hline $\begin{array}{l}\text { Stenorrhynchos hassleri Cogn. } \\
\text { PASSIFLORACEAE }\end{array}$ & A.Laurênio,277 & 7 & $X$ & \\
\hline Passiflora cincinnata Mart. & J.R.R.Cantarelli,16 & 5 & $\mathrm{X}$ & \\
\hline Passiflora foetida $\mathrm{L}$. & A.P.S.Gomes, 213 & 5 & $\mathrm{X}$ & $X$ \\
\hline Passiflora galbana Mart. & M.J.N.Rodal,336 & 5 & $\mathrm{X}$ & \\
\hline Passiflora $\mathrm{sp}$ & A.P.S.Gomes,49 & 5 & $\mathrm{X}$ & \\
\hline \multicolumn{5}{|c|}{ PHYTOLACCACEAE (J.Kallunki - NY; J.W.Novicke - US; S.F.Smith - K) } \\
\hline Gallesia integrifolia (Spreng.) Harms & L.M.Nascimento,36 & 1 & $\mathrm{X}$ & \\
\hline Phytolacca $\mathrm{sp}$ & A.P.S.Gomes, 147 & 4 & $\mathrm{X}$ & \\
\hline Rivina humilis L. & M.F.Sales,609 & 3 & $\mathrm{X}$ & \\
\hline \multicolumn{5}{|l|}{ PIPERACEAE } \\
\hline Peperomia sp1 & A.P.S.Gomes, 139 & 7 & $\mathrm{X}$ & \\
\hline Peperomia sp2 & M.F.Sales,664 & 7 & $\mathrm{X}$ & \\
\hline \multicolumn{5}{|l|}{ PLUMBAGINACEAE } \\
\hline Plumbago scandens L. & S.S.Lira,21 & 3 & $\mathrm{X}$ & \\
\hline \multicolumn{5}{|l|}{ POACEAE (S.A.Revoise - K) } \\
\hline Ichnanthus dasycoleus Tutin & M.F.Sales, 658 & 4 & $\mathrm{X}$ & \\
\hline Ichnanthus nemoralis (Schrad.) Hitchc. \& Chase & L.F.Silva,23 & 4 & $\mathrm{X}$ & \\
\hline Ichnanthus sp & M.J.N.Rodal, 665 & 4 & $\mathrm{X}$ & \\
\hline Panicum sp & E.Inácio, 12 & 4 & $\mathrm{X}$ & \\
\hline \multicolumn{5}{|l|}{ POLYGALACEAE } \\
\hline Polygala albicans A.W.Benn. & A.Laurênio,169 & 2 & & $\mathrm{X}$ \\
\hline Polygala laureola A.St.-Hil. \& Moq. & M.F.Sales, 656 & 3 & $\mathrm{X}$ & \\
\hline Polygala aff. spectabilis DC. & M.J.N.Rodal, 561 & 2 & $\mathrm{X}$ & \\
\hline Polygala sp & M.J.N.Rodal, 683 & 2 & $\mathrm{X}$ & \\
\hline POLYGONACEAE & & & & \\
\hline
\end{tabular}


Tabela 1. (continuação).

\begin{tabular}{|c|c|c|c|c|}
\hline Divisão/Familía/Espécie & Coletor & $\mathrm{H}$ & $\mathrm{T}$ & $\mathrm{EM}$ \\
\hline $\begin{array}{l}\text { Ruprechtia laxiflora } \text { C.A.Mey. } \\
\text { PORTULACACEAE }\end{array}$ & M.F.Sales, 659 & 1 & $X$ & \\
\hline $\begin{array}{l}\text { Talinum paulensis (Jacq.) Willd. } \\
\text { PROTEACEAE }\end{array}$ & M.J.N.Rodal, 651 & 4 & $X$ & \\
\hline $\begin{array}{l}\text { Roupala paulensis Sleum. } \\
\text { RHAMNACEAE }\end{array}$ & M.J.N.Rodal, 657 & 1 & $\mathrm{X}$ & \\
\hline Gouania mollis Reissek & M.J.N.Rodal, 617 & 5 & $\mathrm{X}$ & $\mathrm{X}$ \\
\hline Ziziphus joazeiro Mart. & M.J.N.Rodal, 612 & 1 & $\mathrm{X}$ & $\mathrm{X}$ \\
\hline \multicolumn{5}{|c|}{ RUBIACEAE (D.Zappi - K; E.B.Souza - PEUFR; P.Delprete - NY) } \\
\hline Alibertia sp & A.Laurênio,270 & 2 & & $\mathrm{X}$ \\
\hline Coutarea alba Griseb. & M.C.Tschá,256 & 1 & & $\mathrm{X}$ \\
\hline Coutarea hexandra (Jacq.) K.Schum. & M.F.Sales, 650 & 1 & $X$ & \\
\hline Emmeorrhiza umbellata (Spreng.) K.Schum. & M.J.N.Rodal,661 & 5 & $\mathrm{X}$ & $\mathrm{X}$ \\
\hline Endlichera umbellata C.Presl. & M.J.N.Rodal,339 & 5 & & $\mathrm{X}$ \\
\hline Genipa americana $\mathrm{L}$. & A.Laurênio,263 & 1 & & $X$ \\
\hline Guettarda platypoda DC. & M.J.N.Rodal,647 & 1 & & $\mathrm{X}$ \\
\hline Guettarda sericea Müll.Arg. & M.C.Tschá,137 & 1 & & $\mathrm{X}$ \\
\hline Guettarda $\mathrm{sp}$ & M.J.N.Rodal,618B & 1 & & $X$ \\
\hline Manettia cordifolia Mart. & M.J.N.Rodal, 664 & 5 & $X$ & \\
\hline Mitracarpus scabrellus Benth. & L.M.Nascimento, 13 & 4 & $\mathrm{X}$ & \\
\hline Mitracarpus villosus (Sw.) Cham. \& Schltdl. & M.F.Sales, 667 & 4 & $\mathrm{X}$ & \\
\hline Psychotria cuspidata Bredem. ex Roem. \& Schult. & M.F.Sales,606 & 2 & $\mathrm{X}$ & \\
\hline Psychotria leiocarpa Cham. \& Schlecht. & M.J.N.Rodal, 483 & 2 & $\mathrm{X}$ & \\
\hline Randia armata (Sw.) DC. & M.J.N.Rodal, 355 & 2 & $\mathrm{X}$ & \\
\hline Randia nitida (Humb., Bonpl. \& Kunth) DC. & M.F.Sales, 652 & 2 & $X$ & \\
\hline Richardia grandiflora (Cham. \& Schltdl.) Steud. & L.F.Silva,30 & 4 & $\mathrm{X}$ & \\
\hline Rudgea jacobinensis Müll.Arg. & M.J.N.Rodal, 612 & 1 & $\mathrm{X}$ & \\
\hline \multicolumn{5}{|l|}{ RUTACEAE (J.Kallunki - NY) } \\
\hline Zanthoxylum rhoifolium Lam. & L.M.Nascimento,53 & 1 & $\mathrm{X}$ & \\
\hline \multicolumn{5}{|c|}{ SAPINDACEAE (M.S.Ferrucci - CTES; G.M.Somner - UFRRJ) } \\
\hline Allophylus laevigatus Radlk. & L.M.Nascimento,50 & 1 & $\mathrm{X}$ & \\
\hline Allophyllus quercifolius Radlk. & S.S.Lira,15 & 1 & $\mathrm{X}$ & \\
\hline Cardiospermum corindum $\mathrm{L}$. & M.C.Tschá,253 & 5 & $\mathrm{X}$ & $\mathrm{X}$ \\
\hline Serjania glabata Humb., Bonpl. \& Kunth & M.F.A.Lucena, 170 & 5 & & $\mathrm{X}$ \\
\hline Serjania marginata Casar. & M.F.Sales, 619 & 5 & $\mathrm{X}$ & \\
\hline Urvillea laevis Radlk. & M.F.Sales, 612 & 5 & $\mathrm{X}$ & $X$ \\
\hline Urvillea ulmacea Kunth & A.Laurênio, 183 & 5 & $\mathrm{X}$ & \\
\hline \multicolumn{5}{|l|}{ SAPOTACEAE (T.D.Pennington - K) } \\
\hline Manilkara salzmanii (A.DC.) Lam. & M.F.Sales, 648 & 1 & $\mathrm{X}$ & \\
\hline $\begin{array}{l}\text { Sideroxylon obtusifolium (Roem. \& Schult.) T.D.Penn. } \\
\text { SCROPHULARIACEAE }\end{array}$ & M.Oliveira, 133 & 1 & $X$ & \\
\hline Angelonia biflora Benth. & D.S.Pimentel,64 & 3 & $X$ & \\
\hline Angelonia hookeriana Benth. & A.P.S.Gomes, 43 & 2 & $\mathrm{X}$ & \\
\hline Angelonia sp & L.M.Nascimento,26 & 2 & $\mathrm{X}$ & \\
\hline Scoparia dulcis L. & F.M.O.Villarouco,20 & 2 & $X$ & $X$ \\
\hline
\end{tabular}


Tabela 1. (continuação).

\begin{tabular}{|c|c|c|c|c|}
\hline Divisão/Familía/Espécie & Coletor & $\mathrm{H}$ & $\mathrm{T}$ & $\mathrm{EM}$ \\
\hline \multicolumn{5}{|l|}{ SMILACACEAE } \\
\hline Smilax sp & E.Inácio,100 & 5 & $\mathrm{X}$ & \\
\hline \multicolumn{5}{|c|}{ SOLANACEAE (M.F.Agra - K; M.Nee - NY; S.Knapp - K) } \\
\hline Brunfelsia uniflora (Pohl) D.Don & L.M.Nascimento,9 & 3 & $\mathrm{X}$ & \\
\hline Cestrum obovatum Sendtn. & M.C.Tschá,380 & 2 & $\mathrm{X}$ & \\
\hline Solandra aff. longipes Sendtn. & A.P.S.Gomes, 2 & 1 & $\mathrm{X}$ & \\
\hline Solanum baturitense Huber & M.J.N.Rodal, 488 & 3 & $\mathrm{X}$ & $\mathrm{X}$ \\
\hline Solanum eunoymoides Sendtn. & M.F.Sales,292 & 2 & $\mathrm{X}$ & \\
\hline Solanum gracillimum Sendtn. & M.F.Sales, 607 & 2 & $\mathrm{X}$ & \\
\hline Solanum inaequale Vell. & M.C.Tschá,23 & 2 & $\mathrm{X}$ & \\
\hline Solanum paniculatum L. & M.F.Sales,303 & 2 & $X$ & \\
\hline Solanum stipulaceum Roem. \& Schult. & M.C.Tschá,558 & 2 & $\mathrm{X}$ & \\
\hline Solanum sp1 & E.Inácio,215 & 2 & $\mathrm{X}$ & \\
\hline Solanum $\mathrm{sp} 2$ & M.C.Tschá,151 & 2 & $\mathrm{X}$ & \\
\hline \multicolumn{5}{|l|}{ STERCULIACEAE (C.L.Cristóbal - CTES) } \\
\hline Helicteres macropetala A.St.-Hil. & M.J.N.Rodal,338 & 2 & $\mathrm{X}$ & $\mathrm{X}$ \\
\hline Melochia tomentosa $\mathrm{L}$. & E.Inácio,6 & 4 & $\mathrm{X}$ & \\
\hline \multicolumn{5}{|l|}{ TILIACEAE (A.B.Marcon - PEUFR) } \\
\hline $\begin{array}{l}\text { Prockia crucis P.Browne ex L. } \\
\text { TURNERACEAE }\end{array}$ & \multicolumn{3}{|c|}{ TURNERACEAE } & \\
\hline $\begin{array}{l}\text { Piriqueta } \mathrm{sp} \\
\text { ULMACEAE }\end{array}$ & \multicolumn{3}{|c|}{ ULMACEAE } & \\
\hline $\begin{array}{l}\text { Celtis brasiliensis (Gardner) Planch. } \\
\text { URTICACEAE }\end{array}$ & M.F.Sales, 610 & 1 & $\mathrm{X}$ & \\
\hline $\begin{array}{l}\text { Urera baccifera (L.) Gaudich. } \\
\text { VERBENACEAE (R.W.Sanders - K; S.F.Smith - }\end{array}$ & M.J.N.Rodal,566 & 1 & $\mathrm{X}$ & \\
\hline Lantana caatingensis Moldenh. & M.J.N.Rodal,490 & 2 & $\mathrm{X}$ & \\
\hline Lantana cf. camara L. & D.C.Silva, 82 & 2 & $\mathrm{X}$ & $\mathrm{X}$ \\
\hline Lantana canescens Humb., Bonpl. \& Kunth & M.C.Tschá,119 & 2 & $\mathrm{X}$ & \\
\hline $\begin{array}{l}\text { Lippia schomburgkiana Schauer } \\
\text { VISCACEAE(B.L.Stannard - K) }\end{array}$ & M.F.Sales,345 & 2 & & $\mathrm{X}$ \\
\hline Phoradendron mucronatum Krug \& Urban & M.J.N.Rodal,343 & 7 & & $\mathrm{X}$ \\
\hline Phoradendron cf. piauhyanum Trel. & A.Laurênio,172 & 7 & & $\mathrm{X}$ \\
\hline Phoradendron piperoides (Kunth) Trel. & E.Menezes,35 & 7 & $\mathrm{X}$ & \\
\hline $\begin{array}{l}\text { Phoradendron tunaeforme (DC.) Eichler } \\
\text { VITACEAE(J.Lombardi-HXBH) }\end{array}$ & M.C.Tschá,554 & 7 & & $\mathrm{X}$ \\
\hline Cissus blanchetiana Planch. & M.J.N.Rodal,607 & 5 & $\mathrm{X}$ & \\
\hline Cissus verticillata (L.) Nicolson \& C.E. Jarvis & D.C.Silva,77 & 5 & $\mathrm{X}$ & \\
\hline
\end{tabular}




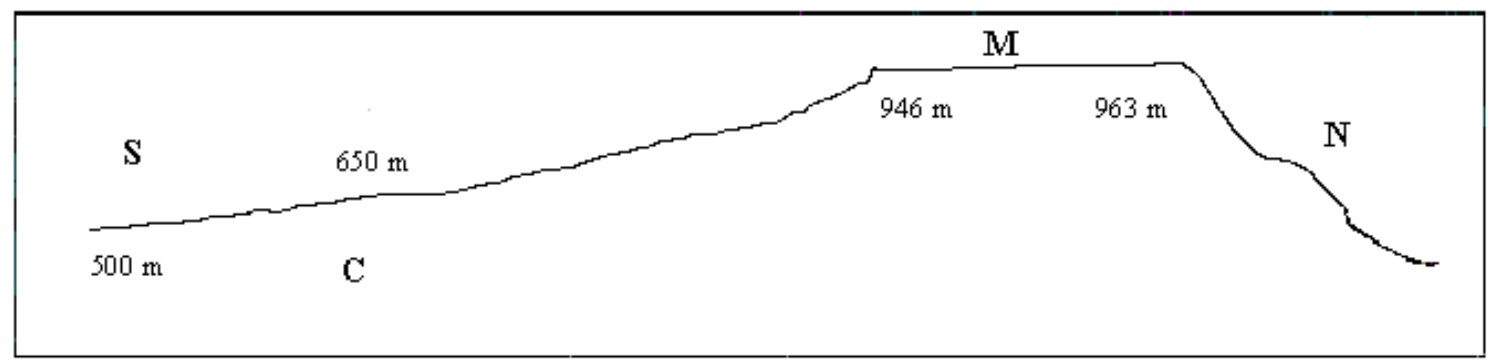

C - calcário com mergutho de $30^{\circ}$ a $40^{\circ}$ para E

$\mathrm{M}$ - topo de meseta em sua maior largura: $800 \mathrm{~m}$

Figura 1. Corte transversal esquemático, no sentido Norte-Sul, mostrando o relevo da Reserva Biológica de Serra Negra, Floresta/Inajá, Pernambuco.

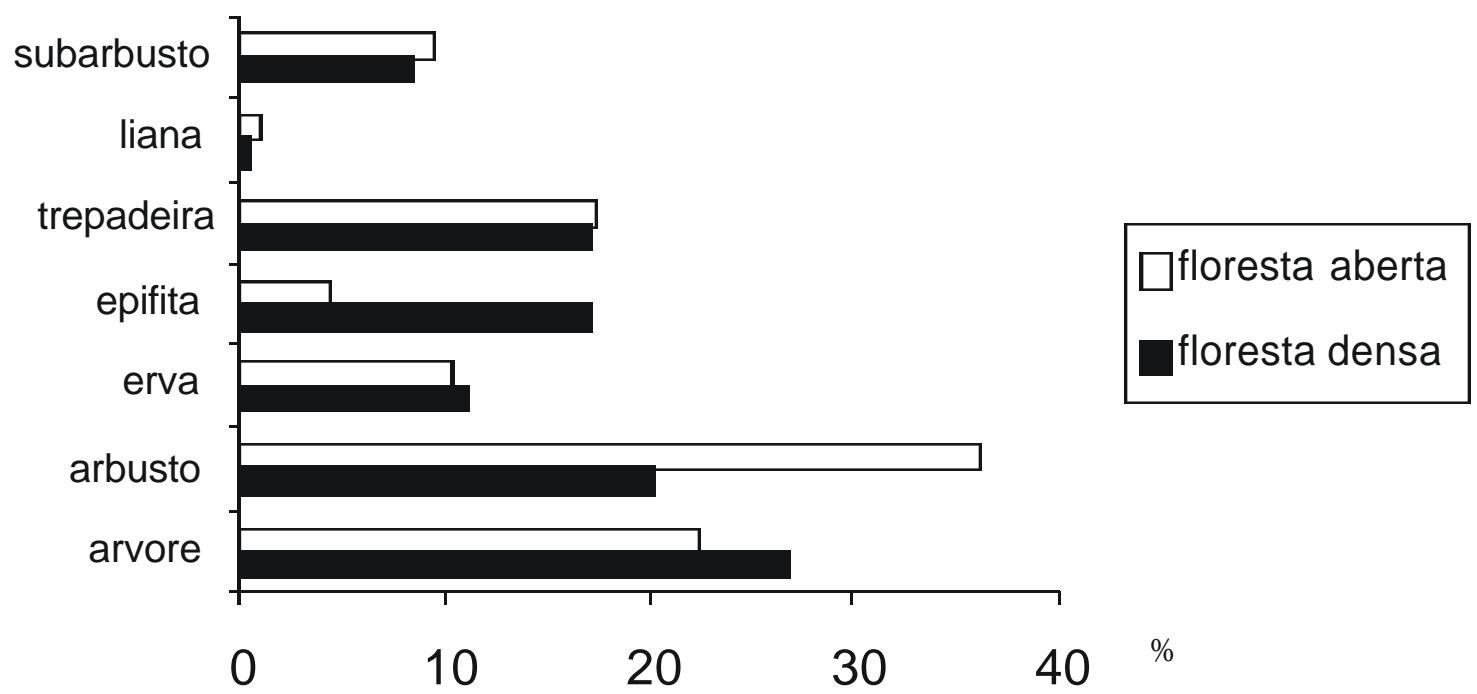

Figura 2. Distribuição percentual do hábito das espécies encontradas nas florestas aberta e densa da Reserva Biológica de Serra Negra, Floresta/Inajá, Pernambuco.

localizadas no domínio do semi-árido e rodeadas por VCE.

As famílias com maior número de espécies no componente arbóreo do topo foram Myrtaceae com 12 espécies, seguida por Mimosaceae (sete) e Malpighiaceae e Apocynaceae (quatro cada). Como árvores mais conspícuas ocorrem: Manilkara salzmanii (Sapotaceae), Trichilia emarginata
(Meliaceae), Albizia polycephala (Mimosaceae), Gallesia integrifolia (Phytolaccaceae), Myrcia fallax, $M$. multiflora (Myrtaceae) e Terminalia brasiliensis (Combretaceae). Ainda nesse componente, deve-se citar uma espécie nova de Malpighiaceae, Bunchosia pernambucana, e que a maioria das espécies não ocorreu na floresta aberta (tabela 1). 


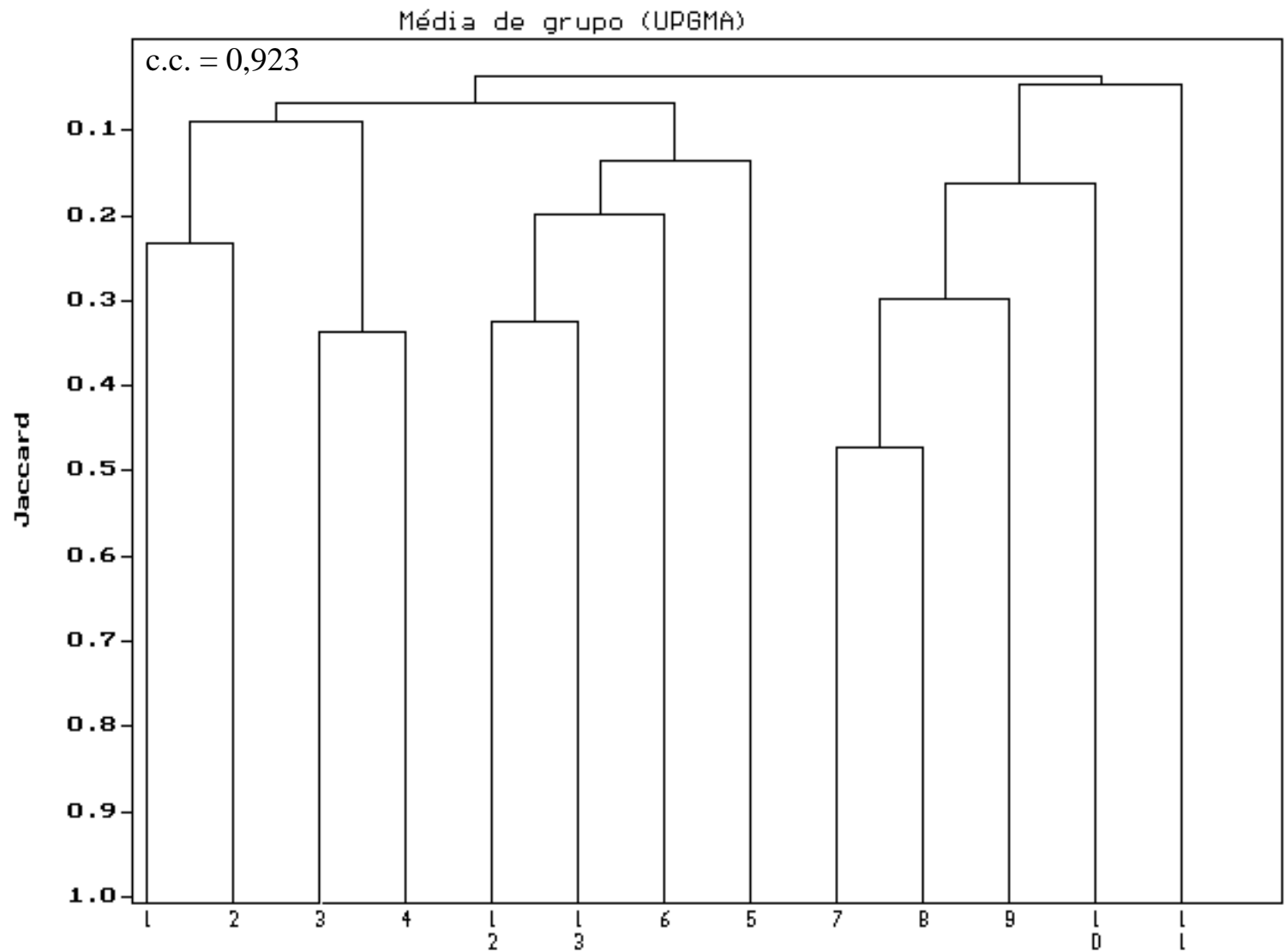

Figura 3. Similaridade florística entre as duas áreas estudadas e outras formações vegetacionais do semi-árido nordestino. Vegetação Caducifólia Espinhosa - caatinga (1/2 - Rodal, 1992; 3/4 - Ferraz et al., 1998), Floresta serrana - brejo de altitude (5/6 - Ferraz et al., 1998); vegetação caducifólia não espinhosa e áreas afins (7/8/9 - Araújo et al., 1998; 10 - Oliveira et al., 1997), Vegetação arbustiva perenifólia sobre chapadas sedimentares (Rodal et al. 1998a), áreas estudadas (12 - floresta densa; 13 - floresta aberta).

A importância de Myrtaceae também foi registrada por Ferraz et al. (1998) na floresta serrana de Triunfo, estado de Pernambuco, e em florestas da costa atlântica brasileira (Peixoto \& Gentry, 1990), sendo considerada por Siqueira (1994) como bastante comum no domínio attântico.

Das Myrtaceae encontradas no topo, algumas como Eugenia biflora e Myrcia fallax têm ampla distribuição nas florestas neotropicais ocorrendo desde a Mesoamérica, Antilhas e América do Sul (McVaugh, 1963; 1969; 1989); Eugenia punicifolia é frequiente na América do Sul; Myrcia multiflora e Myrciaria floribunda são citadas no escudo das Guayanas (McVaugh, 1969) e florestas ciliares como a da bacia hidrográfica do rio Tibagi, Paraná (Dias et al., 1998 e Silva et al., 1992); Calyptranthes multiflora ocorre no escudo das Guayanas (McVaugh, 1969); Eugenia lambertiana var. hispidula no Pará (McVaugh, 1969); Calyptranthes dardanoi tem citação para a zona da mata úmida de Pernambuco (Mattos, 1990).

Dentre as Leguminosae (lato sensu), Inga thibaudiana tem distribuição ampla nos neotrópicos (Pennington, 1997) e é encontrada na Bahia, segundo Lewis (1987), na mata higrófila, restinga, capoeira e plantações de cacau. O contrário ocorre com Albizia polycephala e Acacia riparia, também amplamente distribuídas nas florestas da 
América do Sul tropical (Ducke, 1949), mas registradas em áreas de caatinga, mata de cipó e mata mesófila (Lewis, 1987). Por outro lado, espécies como Parapiptadenia zehntneri, Senna macranthera var. pudibunda e var. micans, são referidas apenas em áreas mais abertas, como VCE, VCNE e cerrado do nordeste brasileiro, especialmente no semi-árido (Irwin \& Barneby, 1982; Lima \& Lima, 1984). Há ainda algumas como Mimosa bimucronata, citada na flora do Rio de Janeiro (Barroso, 1965), mata de várzea da floresta estacional paulista (Metzger et al., 1998) e presente em áreas perturbadas da mata costeira à caatinga, na Bahia (Lewis, 1987).

Entre as Sapotaceae, taxon bastante comum no domínio amazônico (Pennington, 1990) ocorrem apenas 2 espécies: Manilkara salzmanii, com distribuição mais atlântica e bastante comum nas florestas úmidas entre Alagoas e Rio de Janeiro e Sideroxylon obtusifolium, citada desde o México até a Argentina, especialmente em áreas mais secas (Pennington, 1990).

Vale ressaltar o registro de apenas uma Lauraceae e Melastomataceae, comuns no domínio atlântico segundo Joly et al. (1991) e Leitão-Filho (1993). Cabe destacar que Ocotea duckey (Lauraceae) e comumente citada em levantamentos da mata de tabuleiro nordestina (Barbosa, 1996).

Entre as demais espécies: Tabebuia impetiginosa (Bignoniaceae) ocorre nas florestas neotropicais (Gentry, 1992); Schoepfia brasiliensis (Olacaceae) é comum nas florestas neotropicais desde a Argentina até as Guyanas (Sleumer, 1984); Tabebuia serratifolia (Bignoniaceae) ocorre nas florestas úmidas entre Alagoas e Rio de Janeiro e na floresta amazônica (Gentry, 1992; Pinto \& Oliveira-Filho, 1999); Urera baccifera é comum nas florestas pluviais montana e submontana da Floresta Atlântica (Rizzini, 1979); Terminalia brasiliensis é citada na floresta de Araucária do sul do Brasil (Sil- va et al., 1997) ocorrendo ainda nas florestas ciliares na cadeia do Espinhaço na serra do Ambrósio em Minas Gerais (Pirani et al., 1988); Trichilia emarginata é referida para as florestas da Bahia, Minas Gerais e São Paulo (Pennington et al., 1981). Entre as Euphorbiaceae, Maprounea guianensis var. guianensis representa um taxon amazônico (Senna, 1984) enquanto Sebastiania brasiliensis, presente no centro-oeste (Ratter et al., 1988), é bastante freqüente na Argentina, Paraguai, Bolívia e Uruguai (O’Donell \& Lourteig, 1942).

Giulietti \& Pirani (1988) observaram que espécies de ampla distribuição geralmente constituem grande parte da flora de diversas áreas. Avaliando as espécies das famílias com maior riqueza no componente arbóreo do topo da RBSN, e de algumas espécies que mais se destacaram pelo seu maior porte/freqüência, verifica-se a presença de elementos amazônicos e atlânticos.

Essa distribuição, hoje tão particular, pode ser explicada pelo fato de que no quaternário tardio, entre 200.000 e 10.000 anos atrás, o clima mundial foi determinado por ciclos glaciais, onde o clima era mais frio que atualmente e ciclos interglaciais, com condições mais quentes que na atualidade (Houghton \& Woodell, 1989). Prado \& Gibbs (1993) observam que poucos estudos têm sido realizados buscando compreender a influência das mudanças climáticas do Pleistoceno na expansão da vegetação decídua e semi-decídua na América do Sul e que muitas espécies lenhosas, que estariam envolvidos nos processos de migrações cíclicas do Pleistoceno, são membros de comunidades florestais deciduais tropicais e subtropicais, tais como a caatinga e algumas matas semidecíduas de São Paulo e Paraná, entre outras.

Considerando o sub-bosque da floresta densa, foram encontrados muitos indivíduos de outra espécie inédita, o subarbusto Ctenanthe pickeliana (Marantaceae), sendo observadas 
apenas duas espécies de Psychotria (Rubiaceae), taxon citado por Joly et al. (1991) como freqüente no estrato arbustivo da Floresta Atlântica. $P$. cuspidata e $P$. leiocarpa têm distribuições distintas, a primeira amazônica e a segunda bastante comum no Paraguai (http:// mobot.mobot.org). As famílias com maior número de espécies no estrato herbáceo e subarbustivo foram Asteraceae (nove), Malvaceae (seis) e Commelinaceae (cinco). Entre as Bromeliaceae, Portea leptantha tem distribuição centrada nas florestas úmidas nos estados da Paraíba e Pernambuco enquanto Tillandsia juncea e $T$. polystachia são citadas em florestas da América Central e florestas neotropicais, respectivamente (Lyman \& Downs, 1977; 1979).

As famílias com maior número de espécies no estrato arbóreo da floresta aberta foram Mimosaceae e Rubiaceae com sete e cinco espécies, respectivamente (tabela 1). No estrato arbustivo, destacaram-se Mimosaceae (com oito espécies), Caesalpiniaceae e Fabaceae (cinco cada) e Euphorbiaceae (quatro). Exceto por Rubiaceae, as famílias com maior riqueza de espécies no componente arbustivo-arbóreo, foram às mesmas da maioria dos levantamentos quantitativos nos diferentes tipos caducifólios do semi-árido nordestino (Araújo et al., 1995; Oliveira et al., 1997; Ferraz et al., 1998). Por seu maior porte e frequiência, citam-se: Acacia bahiensis, Caesalpinia pyramidalis, Chloroleucon foliolosum, Parapiptadenia zenhtneri e Pithecellobium diversifolium.

Com relação a distribuição das leguminosas lenhosas verifica-se uma padrão bastante variado. Mimosa arenosa tem registro no escudo das Guayanas (Irwin, 1966). Platymiscium floribundum é citada na flora da Guanabara (Barroso, 1965) e em diversos pontos da mata higrófila e capoeira da Bahia (Lewis, 1987). Senna pendula var. dolichandra, é citada na localidade Pedra Azul, Minas Gerais (Irwin \& Barneby, 1982) e em terrenos abandonados de cultivo em áreas de caatinga, na Bahia (Lewis, 1987). Outras por sua vez, têm distribuição centrada dentro dos limites do semi-árido nordestino como: Caesalpinia pyramidalis, Dalbergia catingicola, Piptadenia stipulacea (Ducke, 1953 e Lewis, 1987).

A ocorrência de Rubiaceae no componente lenhoso dos levantamentos do semi-árido é registrada apenas em áreas da VCE onde há maior disponibilidade hídrica, como São José do Belmonte e Caruaru, ambas no estado de Pernambuco (Tavares et al., 1969 e AlcoforadoFilho et al., prelo) e Barbalha no estado do Ceará (Tavares et al., 1974).

Se por um lado, Leguminosae e Rubiaceae e Euphorbiaceae foram importantes no componente lenhoso, a última família ocorreu ainda com representantes subarbustivos, herbáceos e trepadeiras. Phyllanthus cf. amarus é uma erva daninha pantropical, nativa dos neotrópicos (Gilliespie, 1993); Tragia volubilis ocorre em áreas quentes de toda América tropical (Mulgura, 1991); Tragia cf. alienta é citada na província paranaense, abrangendo parte do Argentina, Bolivia e Paraguay (Spichiger \& Ramella, 1990) e Dalechampia olfersiana é citada por Webster (1991) como ocorrente na vegetação estacional de Minas Gerais.

Entre as ervas e trepadeiras, Fabaceae teve maior número de espécies (cinco), seguida por Asteraceae, Commelinaceae e Sapindaceae (três espécies cada), enquanto no estrato epífitico predominaram representantes de Viscaceae (três espécies ).

Os resultados encontrados sugerem que as florestas densa e aberta apresentam distintos padrões de riqueza de espécies e proporção de formas de vida. O predomínio de epífitas na floresta densa provavelmente ocorre em função da maior umidade enquanto a maior porcentagem de arbustos na floresta aberta, pode ser explicada pela maior penetração de luz, devido ao dossel ser mais aberto, bem como por se tratar de um ambiente mais seco.

A floresta densa do topo apresentou diversas espécies comuns a florestas mais úmidas. De 
um modo geral, houve registro de elementos florísticos tanto do domínio amazônico quanto do atlântico. A presença dessas espécies em uma floresta completamente isolada em pleno domínio do semi-árido indica tratar-se de uma área importante em termos de fitodiversidade e merecedora de estudos adicionais que permitam compreender suas relações florísticas, especialmente no que diz respeito a Floresta Atlântica nordestina. A floresta aberta, por sua vez, situada na escarpa meridional, apresentou diversas espécies comuns à VCE, algumas das quais com distribuição restrita aos diferentes tipos caducifólios do semi-árido nordestino enquanto outras têm ocorrência em diferentes pontos da região neotropical.

A riqueza de espécies e a presença plantas com padrões de distribuição acima comentados indica que a RBSN é uma área importante para a fitodiversidade, sendo considerada área de extrema importância biológica para o bioma caatinga (http://www.biodiversitas.org/caatinga/).

\section{Referências bibliográficas}

Alcoforado-Filho, F.G.; Sampaio, E.V.S.B. \& Rodal, M.J.N. prelo. Florística e fitossociologia de um remanescente de vegetação caducifólia espinhosa arbórea em Caruaru, Pernambuco. Acta Botanica Brasilica.

Andrade, G.O. de. 1954. A Serra Negra - uma relíquia geomórfica e higrófita nos tabuleiros pernambucanos. Imprensa Oficial. Recife.

Andrade-Lima, D. de. 1954. Contribution to the study of the flora of Pernambuco, Brazil. Monografia. Universidade Federal Rural de Pernambuco. Recife.

Andrade-Lima, D. de. 1957. Estudos fitogeográficos de Pernambuco. Instituto de Pesquisas Agronômicas de Pernambuco, 50 p. (Publicação, n.2)

Andrade-Lima, D. de. 1961. Tipos de florestas de Pernambuco. Anais da Associação Brasileira de Geógrafos 12: 69-85.

Andrade-Lima, D. de. 1966. Esboço fitoecológico de alguns brejos de Pernambuco. Boletim Técnico IPA, 7p. (Boletim Técnico, n. 8).

Andrade-Lima, D. de. 1973. Traços gerais do agreste de Pernambuco. Pp. 85-88. In: Anais do 23 ${ }^{\text {a Con- }}$ gresso Brasileiro de Botânica. Sociedade Brasileira de Botânica. Recife.

Andrade-Lima, D. de. 1982. Present-day forest refuges in Northeastern Brazil. Pp. 247-251. In: G. T. Prance (Ed.), Biological diversification in the tropics. Columbia University Press, New York.

Andrade-Lima, D. de. 1989. Plantas da caatinga. Academia Brasileira de Ciências, Rio de Janeiro.

Araújo, E.L.; Sampaio, E.V.S.B. \& Rodal, M.J.N. 1995. Composição florística e fitossociológica de três áreas de caatinga. Revista Brasileira de Biologia 55(4): 595-607.

Araújo, F.S.; Sampaio, E.V.S.B.; Rodal, M.J.N. \& Figueiredo, M.A. 1998. Organização comunitária do componente lenhoso de três áreas de carrasco em Nova Oriente - CE. Revista Brasileira de Biologia 58(1): 85-95.

Barbosa, M.R.V.B. 1996. Estudo florístico e fitossociológico da Mata do Buraquinho, remanescente de Mata Atlântica em João Pessoa, PB. Tese de Doutorado. Universidade Estadual de Campinas.. Campinas.

Barroso, G.M. 1965. Leguminosas da Guanabara. Arquivos do Jardim Botânico do Rio de Janeiro 18: 109-177.

Bridson, D. \& Forman, L. 1998. The herbarium handbook. $2^{\mathrm{a}}$ ed. Royal Botanic Gardens Kew, London.

Brummitt, R.F. 1992. Vascular plant families and genera. Royal Botanic Gardens Kew, London.

Brummitt, R.F. \& Powell, C.E. 1992. Authors of plant names. Royal Botanic Gardens /Kew, London.

Cronquist, A. 1981. An integrated system of classification of flowering plants.Columbia University Press, New York.

Dantas, J.R.A. 1980. Mapa geológico do estado de Pernambuco. DNPM. Recife.

Dias, M.C.; Vieira, A.O.S.; Nakajima, J.N.; Pimenta, J.A.\& Lobo, P.C. 1998. Composição florística e fitossociológica do componente arbóreo das florestas ciliares do rio Tibagi, PR. Revista Brasileira de Botânica 21(2): 183-195.

Ducke, A. 1949. Notas sobre a flora neotrópica II. As leguminosas da Amazônia Brasileira. Boletim Técnico do Instituto Agronômico 18:1-248

Ducke, A. 1953. As Leguminosas de Pernambuco e Paraíba. Memórias do Instituto Oswaldo Cruz 51: 417-461.

Ferraz, E.M.N.; Rodal, M.J.N.; Sampaio, E.V.S.B. \& Pereira, R. de C.A. 1998. Composição florística em trechos de vegetação de caatinga e brejo de alti- 
tude na região do Vale do Pajeú, Pernambuco. Revista Brasileira de Botânica 21(1): 7-15.

Font Quer, M.P. 1977. Diccionario de botánica. Lábor., Barcelona.

Gentry, A.H. 1992. Flora neotropica: Tribe Tecomeae (Bignoniaceae). Part. 2. Organization for Flora Neotropica by Hafner Publishing Company (Monograph, 25), New York.

Gilliespie, L.J. 1993. Euphorbiaceae of Guianas: Annotated species checlist and key to the genera. Brittonia 45(1): 56-94.

Giulietti, A.M. \& Pirani, J.R. 1988. Patterns of geographic distribution of some plant species from the Espinhaço Range, Minas Gerais and Bahia. Pp . 39-69. In: W.R. Heyer \& P.E. Vanzoloni (Eds.), Proceedings of a Workshop on Neotropical Distribution Patterns. Academia Brasileira de Ciências, Rio de Janeiro.

Heywood, V.H. 1997. Centres of plant diversity. WWF/IUCN, London.

Houghton, R. \& Woodell, G. 1989. Cambio climático global. Investigación y Ciência 153(1): 8-17

IBAMA. 1989. Unidades de conservação do Brasil: parques nacionais e reservas biológicas. Brasília: Ministério do Interior.

Irwin, H. S. 1966. Contributions to the Botany of Guiana. III. Leguminosae-Mimosoideae. Memoirs of the New York Botanical Garden 15(1): 96-111

Irwin, H.S. \& Barneby, R.C. 1982. The american Cassiinae: A synoptical revision of Leguminosae tribe Cassieae subtribe Cassiinae in the New World. Memoirs of The New York Botanical Garden 35:1-918.

Joly, C.B.; Leitão-Filho, H.F. \& Silva, S.M. 1991. O patrimônio florístico. Pp. 96-128. In: Câmara, I. G., (Ed.), Mata Atlântica. SOS Mata Atlântica, São Paulo.

Kent, M. \& Coker, P. 1992. Vegetation description and analysis: a pratical approach. John Wiley \& Sons, London.

Leitão-Filho, H.F. 1993. Ecologia da Mata Atlântica em Cubatão (SP). EDUSP/UNICAMP, São Paulo.

Lewis, G.P. 1987. Legumes of Bahia. Royal Botanic Gardens Kew, London.

Lima, M.P.M. \& Lima, H.C. 1984. Parapiptadenia Brenan (Leg. Mim.) - Estudo taxonômico das espécies brasileiras. Rodriguesia 36(60): 23-30.

Lyman, B.S. \& Downs, R.J. 1977. Flora neotropica: Tillandsioideae (Bromeliaceae) Part. 2. Organization for Flora Neotropica by Hafner Publishing Company (Monograph, 13), New York.
Lyman, B.S. \& Downs, R.J. 1979. Flora neotropica: Bromelioideae (Bromeliaceae) Part. 3. Organization for Flora Neotropica by Hafner Publishing Company (Monograph, 14), New York.

Lyra, A.L.R.T. 1982. Efeito do relevo na vegetação em duas áreas do município de Brejo da Madre de Deus, Pernambuco. Dissertação de Mestrado. Universidade Federal Rural de Pernambuco. Recife.

Mattos, J.R. 1990. Novidades taxonômicas em Myrtaceae - VI. Loefgrenia - comunicações avulsas de Botânica 99: 1-6.

McVaugh, R. 1963. Flora of Guatemala: Myrtaceae. Fieldiana 24(7): 283-405.

McVaugh, R. 1969. Myrtaceae. In: B. Maguire \& collaborators. The botany of the Guayana Highland - Part VIII. Memoirs of the New York Botanical Garden 18: 55-286.

McVaugh, R. 1989. Myrtaceae Pp. 463-532. In: Dicotyledoneae - Part 2. Flora of the Lesser Antilles, Leeward and Windward Islands.

Metzger, J.P.; Goldenberg, R. \& Bernacci, L.C. 1998. Diversidade e estrutura de fragmentos de mata de várzea e de mata mesófila semi-decídua submontana do rio Jacaré-Pepira (SP). Revista Brasileira de Botânica 21(3): 321-330.

Mulgura, M. E. 1991. Sinopsis del genero Tragia L. (Euphorbiaceae) del Paraguay Candollea 46: 521-532.

O'Donell, C.A \& Lourteig, A. 1942. Hipomaneae Argentinae (Euphorbiaceae). Lilloa 8: 455-592.

Oliveira, A.P. \& Andrade-Lima, D. de. 1948. Serra Negra. Boletim Técnico da Secretária de Agricultura, Indústria e Comércio do estado de Pernambuco. p. 388-397.

Oliveira, M.E.A.; Sampaio, E.V.S.B.; Castro, A.A.J. de F. \& Rodal, M.J.N. 1997. Flora e fitossociologia de uma área de transição carrasco-caatinga de areia em Padre Marcos, Piauí. Naturalia 22(2): 131-150.

Peixoto, A.L. \& Gentry, A.H. 1990. Diversidade e composição florística da mata de tabuleiro na Reserva Florestal de Linhares (Espírito Santo, Brasil). Revista Brasileira de Botânica 13:19-25.

Pennington, T.D. 1990. Flora neotropica: Sapotaceae. Organization for Flora Neotropica by Hafner Publishing Company. (Monograph, 52), New York

Pennington, T.D. 1997. The Genus Inga - Botany. Royal Botanic Gardens Kew, London.

Pennington, T.D.; Styles, B. T. \&. Taylor, D. A. H. 1981. Flora neotropica: Meliaceae. Organization for Flora Neotropica by Hafner Publishing Company. (Monograph, 28), New York.

Pereira, R.C.A.; Lima,V.C.; Silva, R.S.; Silva, S.Z. 1993. 
Lista das espécies arbóreas e arbustivas ocorrentes nos principais "brejos" de altitude de Pernambuco. Instituto de Pesquisas Agronômicas de Pernambuco. Série documentos, 22, Recife.

Pinto, J.R.R. \& Oliveira-Filho, A.T. de 1999. Perfil florístico e estrutura da comunidade arbórea de uma floresta de vale no Parque Nacional da Chapada dos Guimarães. Revista Brasileira de Botânica 22(1): 53-67.

Pirani, J.R.; Giulietti, A.M.; Mello-Silva, R. \& Meguro, M. 1988. Checklist and patterns of geographic distribution of the vegetation of Serra do Ambrósio, Minas Gerais, Brasil. Revista Brasileira de Botânica 17(2): 133-147.

Prado, D.E. \& Gibbs, P.E. 1993. Patterns of species distributions in the dry seasonal forest of South America. Annals Missouri Botanical Garden 80: 902-927.

Ratter, J.A.; Pott, A J.V.; Cunha, C.N. da \& Haridasan, M. 1988. Observations on woody vegetation types in the pantanal and at Corumbá, Brazil. Notes of Royal Botanic Garden of Edinburgh 45(3): 503-525.

Rizzini, C.T. 1979. Tratado de Fitogeografia do Brasil: aspectos sociológicos e florísticos. HUCITEC/ USP, São Paulo.

Rodal, M.J.N. 1992. Fitossociologia da vegetação arbustivo-arbórea em quatro áreas de caatinga em Pernambuco. Tese de Doutorado. Universidade Estadual de Campinas. Campinas.

Rodal, M.J.N., Sales, M.F. de; Mayo, S.J. 1998b. Florestas serranas de Pernambuco: localização e diversidade dos remanescentes dos brejos de altitude. Imprensa Universitária. Universidade Federal Rural de Pernambuco, Recife.

Rodal, M.J.N.; Andrade, K.V. de S.A.; Sales, M.F. de \& Gomes, A.P.S. 1998a. Fitossociologia do componente lenhoso de um refúgio vegetacional no município de Buíque, Pernambuco. Revista Brasileira de Biologia 58(3): 517-526.

Sales, M.F.; Mayo, S.J.; Rodal, M.J.N. 1998. Plantas vasculares das florestas serranas de Pernambuco - Um checklist da flora ameaçada dos brejos de altitude. Imprensa Universitária. Universidade Federal Rural de Pernambuco, Recife.

Senna. L M. 1984. Maprounea Aubl. (Euphorbiaceae) - Considerações taxonômicas das espécies sulamericanas. Rodriguesia 36(60): 51-78.

Shepherd, G.J. 1995. FITOPAC: Manual do usuário. Departamento de Botânica. Universidade Estadual de Campinas, Campinas.

Silva, J.A.S.; Salomão, A. N.; Gripp, A. \& Leite, E.J.
1997. Phytosociological survey in Brazilian forest genetic of Caçador. Plant Ecology 133(1): 1-11.

Silva, S.M; Silva, F.C.; Vieira, A.O.S; Nakajima, J.N.; Pimenta, J.A.; Colli, S. 1992. Composição florística e fitossociologia do componente arbóreo das florestas ciliares da bacia do rio Tibagi, Paraná: 2. Várzea do rio Bitumirim de Ipiranga, PR. Revista do Instituto Florestal 4:192-206.

Siqueira, M.F. de. 1994. Análise florística e ordenação de espécies arbóreas da Mata Atlântica através de dados binários. Tese de Mestrado. Universidade Estadual de Campinas. Campinas.

Sleumer, B. 1984. Flora neotropica: Olacaceae. Organization for Flora Neotropica by Hafner Publishing Company (Monograph, 35), New York.

Souza, M. J.N. de; Martins, M.L.R.; Soares, Z.M.L.; Freitas-Filho, M.R. de; Almeida, M.A.G. de, Pinheiro, F.S. de A.; Sampaio, M.A.B.; Carvalho, G.M.B.S.; Soares, A.M.L.; Gomes, E.C.B. \& Silva, R.A. 1994. Redimensionamento da região semi-árida do Nordeste do Brasil. In: Conferência Nacional e Seminário Latino-Americano de Desertificação. Fundação Esquel do Brasil, Fortaleza.

Spichiger, R. \& Ramella, L. 1990. Notulae ad Floram paraquaiensem. Candollea 45(1): 35-45

Tavares, S.; Paiva, F.A.V.; Tavares, E.J. de S. \& Lima, J.L.S. de. 1974. Inventário florestal do Ceará. Estudo preliminar das matas remanescentes do município de Barbalha. Boletim de Recursos Naturais 12(2):20-46.

Tavares, S.; Paiva, F.A.V.; Tavares, E.J. de S.; Lima, J.L.S. de. \& Carvalho, G.H. 1969. Inventário florestal de Pernambuco. Estudo preliminar das matas remanescentes do município de São José do Belmonte. Boletim de Recursos Naturais 7(1/ 4):113-139.

Tryon, R. \& Tryon, A.F. 1982. Ferns and allied plants with special reference to tropical America. Springer - Verlag, New York.

Valentim, J.L. 2000. Ecologia numérica: uma introdução a análise multivariada de dados: Editora Interciência, Rio de Janeiro.

Vasconcelos-Sobrinho, J. 1949. As regiões naturais de Pernambuco, o meio e a civilização. Livraria Freitas Bastos, Rio de Janeiro.

Vitt, D.H. 1984. Classification of Bryopsida Pp. 696759. In: R. N. Schuster (Ed.), Manual of Bryology Hatpori Botany Labotatory Nichigan, Mijazaki.

Webster, G.L. 1991. A synopsis of the Neotropical species of Dalechampia (Euphorbiaceae). Journal of the Linnean Society 105: 137-177. 